\title{
Gauged baryon and lepton numbers in supersymmetry with a $125 \mathrm{GeV}$ Higgs
}

\author{
Tai-Fu Feng ${ }^{a *}$, Shu-Min Zhao ${ }^{a \dagger}$, Hai-Bin Zhang ${ }^{a, b}$, Yin-Jie Zhang ${ }^{a}$, Yu-Li Yan ${ }^{a}$ \\ ${ }^{a}$ Department of Physics, Hebei University, Baoding, 071002, China \\ ${ }^{b}$ Department of Physics, Dalian University of Technology, Dalian, 116024, China
}

\begin{abstract}
Assuming that the Yukawa couplings between the Higgs and exotic quarks cannot be ignored, we analyze the signals of decay channels $h \rightarrow \gamma \gamma$ and $h \rightarrow V V^{*}(V=Z, W)$ with the Higgs mass around $125 \mathrm{GeV}$ in a supersymmetric extension of the standard model where baryon and lepton numbers are local gauge symmetries. Adopting some assumptions on relevant parameter space, we can account for the experimental data on Higgs from ATLAS and CMS naturally.
\end{abstract}

PACS numbers: 14.80.Cp, $12.15 \mathrm{Hh}$

Keywords: Supersymmetry, Baryon and Lepton numbers, Higgs

\footnotetext{
* email:fengtf@hbu.edu.cn

† email:smzhao@hbu.edu.cn
} 


\section{INTRODUCTION}

The main destination of the Large Hadron Collider (LHC) is to understand the origin of the electroweak symmetry breaking, and searches the neutral Higgs predicted by the standard model (SM) and its various extensions. Recently, ATLAS and CMS have reported significant excess events which are interpreted probably to be related to the neutral Higgs with mass $m_{h_{0}} \sim 124-126 \mathrm{GeV}[1,2]$. This implies that the Higgs mechanism to break electroweak symmetry possibly has a solid experimental cornerstone.

As the simplest soft broken supersymmetry theory, the minimal supersymmetric extension of the standard model (MSSM) [3] has drawn the attention from physicist for a long time. Furthermore, Broken baryon number (B) can explain the origin of the matter-antimatter asymmetry in the Universe naturally. Since heavy majorana neutrinos contained in the seesaw mechanism can induce the tiny neutrino masses [4] to explain the neutrino oscillation experiment, lepton number (L) is also expected to be broken. Ignoring Yukawa couplings between Higgs doublets and exotic quarks, the authors of literature [5, 6] investigate the predictions for the mass and decays of the lightest CP-even Higgs in a minimal local gauged B and L supersymmetric extension of the SM which is named BLMSSM. Since the new quarks are vector-like, one obtains that their masses can be above $500 \mathrm{GeV}$ without assuming large couplings to the Higgs doublets in this model. Therefore, there are no Landau poles for the Yukawa couplings here. Additionally, literature[7] also examines two extensions of the SM where $B$ and $L$ are spontaneously broken gauge symmetries around $\mathrm{TeV}$ scale. Assuming that the Yukawa couplings between Higgs and exotic quarks cannot be ignored here, we investigate the lightest CP-even Higgs decay channels $h \rightarrow \gamma \gamma, h \rightarrow V V^{*}(V=Z, W)$ in the BLMSSM.

Our presentation is organized as follows. In section II, we briefly summarize the main ingredients of the BLMSSM, then present the mass squared matrices for neutral scalar sectors and the mass matrices for exotic quarks, respectively. We discuss the corrections on the mass squared matrix of CP-even Higgs from exotic fields in section III, and present the decay widths for $h^{0} \rightarrow \gamma \gamma, V V^{*}(V=Z, W)$ in section IV. The numerical analyses are given in section $\mathrm{V}$, and our conclusions are summarized in section VI. 


\section{A SUPERSYMMTRIC EXTENSION OF THE SM WHERE B AND L ARE LOCAL GAUGE SYMMETRIES}

When B and L are local gauge symmetries, one can enlarge the local gauge group of the SM to $S U(3)_{C} \otimes S U(2)_{L} \otimes U(1)_{Y} \otimes U(1)_{B} \otimes U(1)_{L}$. In the supersymmetric extension of the SM proposed in Ref. [5, 6], the exotic superfields include the new quarks $\hat{Q}_{4} \sim\left(3,2,1 / 6, B_{4}, 0\right)$, $\hat{U}_{4}^{c} \sim\left(\overline{3}, 1,-2 / 3,-B_{4}, 0\right), \hat{D}_{4}^{c} \sim\left(\overline{3}, 1,1 / 3,-B_{4}, 0\right), \hat{Q}_{5}^{c} \sim\left(\overline{3}, 2,-1 / 6,-\left(1+B_{4}\right), 0\right)$, $\hat{U}_{5} \sim\left(3,1,2 / 3,1+B_{4}, 0\right), \hat{D}_{5} \sim\left(3,1,-1 / 3,1+B_{4}, 0\right)$, and the new leptons $\hat{L}_{4} \sim\left(1,2,-1 / 2,0, L_{4}\right), \hat{E}_{4}^{c} \sim\left(1,1,1,0,-L_{4}\right), \hat{N}_{4}^{c} \sim\left(1,1,0,0,-L_{4}\right), \hat{L}_{5}^{c} \sim$ $\left(1,2,1 / 2,0,-\left(3+L_{4}\right)\right), \hat{E}_{5} \sim\left(1,1,-1,0,3+L_{4}\right), \hat{N}_{5} \sim\left(1,1,0,0,3+L_{4}\right)$ to cancel the $B$ and $L$ anomalies. The 'brand new' Higgs superfields $\hat{\Phi}_{B} \sim(1,1,0,1,0)$ and $\hat{\varphi}_{B} \sim(1,1,0,-1,0)$ acquire nonzero vacuum expectation values (VEVs) to break Baryon number spontaneously. Meanwhile, nonzero VEVs of $\Phi_{B}$ and $\phi_{B}$ also induce the large masses for exotic quarks. In addition, the superfields $\hat{\Phi}_{L} \sim(1,1,0,0,-2)$ and $\hat{\varphi}_{L} \sim(1,1,0,0,2)$ acquire nonzero VEVs to break Lepton number spontaneously. In order to avoid stability for the exotic quarks, the model also includes the superfields $\hat{X} \sim$ $\left(1,1,0,2 / 3+B_{4}, 0\right), \hat{X}^{\prime} \sim\left(1,1,0,-\left(2 / 3+B_{4}\right), 0\right)$. Actually, the lightest one can be a dark matter candidate. The superpotential of the model is written as

$$
\mathcal{W}_{B L M S S M}=\mathcal{W}_{M S S M}+\mathcal{W}_{B}+\mathcal{W}_{L}+\mathcal{W}_{X}
$$

where $\mathcal{W}_{M S S M}$ is superpotential of the MSSM, and

$$
\begin{aligned}
\mathcal{W}_{B}= & \lambda_{Q} \hat{Q}_{4} \hat{Q}_{5}^{c} \hat{\Phi}_{B}+\lambda_{U} \hat{U}_{4}^{c} \hat{U}_{5} \hat{\varphi}_{B}+\lambda_{D} \hat{D}_{4}^{c} \hat{D}_{5} \hat{\varphi}_{B}+\mu_{B} \hat{\Phi}_{B} \hat{\varphi}_{B} \\
& +Y_{u_{4}} \hat{Q}_{4} \hat{H}_{u} \hat{U}_{4}^{c}+Y_{d_{4}} \hat{Q}_{4} \hat{H}_{d} \hat{D}_{4}^{c}+Y_{u_{5}} \hat{Q}_{5}^{c} \hat{H}_{d} \hat{U}_{5}+Y_{d_{5}} \hat{Q}_{5}^{c} \hat{H}_{u} \hat{D}_{5}, \\
\mathcal{W}_{L}= & Y_{e_{4}} \hat{L}_{4} \hat{H}_{d} \hat{E}_{4}^{c}+Y_{\nu_{4}} \hat{L}_{4} \hat{H}_{u} \hat{N}_{4}^{c}+Y_{e_{5}} \hat{L}_{5}^{c} \hat{H}_{u} \hat{E}_{5}+Y_{\nu_{5}} \hat{L}_{5}^{c} \hat{H}_{d} \hat{N}_{5} \\
& +Y_{\nu} \hat{L} \hat{H}_{u} \hat{N}^{c}+\lambda_{N^{c}} \hat{N}^{c} \hat{N}^{c} \hat{\varphi}_{L}+\mu_{L} \hat{\Phi}_{L} \hat{\varphi}_{L}, \\
\mathcal{W}_{X}= & \lambda_{1} \hat{Q} \hat{Q}_{5}^{c} \hat{X}+\lambda_{2} \hat{U}^{c} \hat{U}_{5} \hat{X}^{\prime}+\lambda_{3} \hat{D}^{c} \hat{D}_{5} \hat{X}^{\prime}+\mu_{X} \hat{X} \hat{X}^{\prime} .
\end{aligned}
$$

In the superpotential above, the exotic quarks obtain TeV scale masses after $\Phi_{B}, \varphi_{B}$ acquire nonzero VEVs, and the nonzero $\mathrm{VEV}$ of $\varphi_{L}$ implements the seesaw mechanism for the tiny 
neutrino masses. Correspondingly, the soft breaking terms are generally given as

$$
\begin{aligned}
\mathcal{L}_{\text {soft }}= & \mathcal{L}_{\text {soft }}^{M S S M}-\left(m_{\tilde{N}^{c}}^{2}\right)_{I J} \tilde{N}_{I}^{c *} \tilde{N}_{J}^{c}-m_{\tilde{Q}_{4}}^{2} \tilde{Q}_{4}^{\dagger} \tilde{Q}_{4}-m_{\tilde{U}_{4}}^{2} \tilde{U}_{4}^{c *} \tilde{U}_{4}^{c}-m_{\tilde{D}_{4}}^{2} \tilde{D}_{4}^{c *} \tilde{D}_{4}^{c} \\
& -m_{\tilde{Q}_{5}}^{2} \tilde{Q}_{5}^{c \dagger} \tilde{Q}_{5}^{c}-m_{\tilde{U}_{5}}^{2} \tilde{U}_{5}^{*} \tilde{U}_{5}-m_{\tilde{D}_{5}}^{2} \tilde{D}_{5}^{*} \tilde{D}_{5}-m_{\tilde{L}_{4}}^{2} \tilde{L}_{4}^{\dagger} \tilde{L}_{4}-m_{\tilde{\nu}_{4}}^{2} \tilde{\nu}_{4}^{c *} \tilde{\nu}_{4}^{c} \\
& -m_{\tilde{E}_{4}}^{2} \tilde{e}_{4}^{c *} \tilde{e}_{4}^{c}-m_{\tilde{L}_{5}}^{2} \tilde{L}_{5}^{c \dagger} \tilde{L}_{5}^{c}-m_{\tilde{\nu}_{5}}^{2} \tilde{\nu}_{5}^{*} \tilde{\nu}_{5}-m_{\tilde{E}_{5}}^{2} \tilde{e}_{5}^{*} \tilde{e}_{5}-m_{\Phi_{B}}^{2} \Phi_{B}^{*} \Phi_{B} \\
& -m_{\varphi_{B}}^{2} \varphi_{B}^{*} \varphi_{B}-m_{\Phi_{L}}^{2} \Phi_{L}^{*} \Phi_{L}-m_{\varphi_{L}}^{2} \varphi_{L}^{*} \varphi_{L}-\left(m_{B} \lambda_{B} \lambda_{B}+m_{L} \lambda_{L} \lambda_{L}+h . c .\right) \\
& +\left\{A_{u_{4}} Y_{u_{4}} \tilde{Q}_{4} H_{u} \tilde{U}_{4}^{c}+A_{d_{4}} Y_{d_{4}} \tilde{Q}_{4} H_{d} \tilde{D}_{4}^{c}+A_{u_{5}} Y_{u_{5}} \tilde{Q}_{5}^{c} H_{d} \tilde{U}_{5}+A_{d_{5}} Y_{d_{5}} \tilde{Q}_{5}^{c} H_{u} \tilde{D}_{5}\right. \\
& \left.+A_{B Q} \lambda_{Q} \tilde{Q}_{4} \tilde{Q}_{5}^{c} \Phi_{B}+A_{B U} \lambda_{U} \tilde{U}_{4}^{c} \tilde{U}_{5} \varphi_{B}+A_{B D} \lambda_{D} \tilde{D}_{4}^{c} \tilde{D}_{5} \varphi_{B}+B_{B} \mu_{B} \Phi_{B} \varphi_{B}+h . c .\right\} \\
& +\left\{A_{e_{4}} Y_{e_{4}} \tilde{L}_{4} H_{d} \tilde{E}_{4}^{c}+A_{N_{4}} Y_{N_{4}} \tilde{L}_{4} H_{u} \tilde{N}_{4}^{c}+A_{e_{5}} Y_{e_{5}} \tilde{L}_{5}^{c} H_{u} \tilde{E}_{5}+A_{N_{5}} Y_{\nu_{5}} \tilde{L}_{5}^{c} H_{d} \tilde{N}_{5}\right. \\
& \left.+A_{N} Y_{N} \tilde{L} H_{u} \tilde{N}^{c}+A_{N^{c}} \lambda_{N^{c}} \tilde{N}^{c} \tilde{N}^{c} \varphi_{L}+B_{L} \mu_{L} \Phi_{L} \varphi_{L}+h . c .\right\} \\
& +\left\{A_{1} \lambda_{1} \tilde{Q} \tilde{Q}_{5}^{c} X+A_{2} \lambda_{2} \tilde{U}^{c} \tilde{U}_{5} X^{\prime}+A_{3} \lambda_{3} \tilde{D}^{c} \tilde{D}_{5} X^{\prime}+B_{X} \mu_{X} X X^{\prime}+h . c .\right\}
\end{aligned}
$$

where $\mathcal{L}_{\text {soft }}^{M S S M}$ is soft breaking terms of the MSSM, $\lambda_{B}, \lambda_{L}$ are gauginos of $U(1)_{B}$ and $U(1)_{L}$, respectively. After the $S U(2)_{L}$ doublets $H_{u}, H_{d}$ and $S U(2)_{L}$ singlets $\Phi_{B}, \varphi_{B}, \Phi_{L}, \varphi_{L}$ acquire the nonzero VEVs $v_{u}, v_{d}, v_{B}, \bar{v}_{B}$, and $v_{L}, \bar{v}_{L}$,

$$
\begin{aligned}
& H_{u}=\left(\begin{array}{c}
H_{u}^{+} \\
\frac{1}{\sqrt{2}}\left(v_{u}+H_{u}^{0}+i P_{u}^{0}\right)
\end{array}\right), \\
& H_{d}=\left(\begin{array}{c}
\frac{1}{\sqrt{2}}\left(v_{d}+H_{d}^{0}+i P_{d}^{0}\right) \\
H_{d}^{-}
\end{array}\right), \\
& \Phi_{B}=\frac{1}{\sqrt{2}}\left(v_{B}+\Phi_{B}^{0}+i P_{B}^{0}\right) \\
& \varphi_{B}=\frac{1}{\sqrt{2}}\left(\bar{v}_{B}+\varphi_{B}^{0}+i \bar{P}_{B}^{0}\right) \\
& \Phi_{L}=\frac{1}{\sqrt{2}}\left(v_{L}+\Phi_{L}^{0}+i P_{L}^{0}\right) \\
& \varphi_{L}=\frac{1}{\sqrt{2}}\left(\bar{v}_{L}+\varphi_{L}^{0}+i \bar{P}_{L}^{0}\right)
\end{aligned}
$$

the local gauge symmetry $S U(2)_{L} \otimes U(1)_{Y} \otimes U(1)_{B} \otimes U(1)_{L}$ is broken down to the electromagnetic symmetry $U(1)_{e}$, where

$$
G^{ \pm}=\cos \beta H_{d}^{ \pm}+\sin \beta H_{u}^{ \pm}
$$


denotes the charged Goldstone boson, and

$$
\begin{aligned}
& G^{0}=\cos \beta P_{d}^{0}+\sin \beta P_{u}^{0}, \\
& G_{B}^{0}=\cos \beta_{B} P_{B}^{0}+\sin \beta_{B} \bar{P}_{B}^{0}, \\
& G_{L}^{0}=\cos \beta_{L} P_{L}^{0}+\sin \beta_{L} \bar{P}_{L}^{0}
\end{aligned}
$$

denote the neutral Goldstone bosons, respectively. Here $\tan \beta=v_{u} / v_{d}, \tan \beta_{B}=\bar{v}_{B} / v_{B}$, and $\tan \beta_{L}=\bar{v}_{L} / v_{L}$. Correspondingly, the physical neutral pseudoscalar fields are

$$
\begin{aligned}
& A^{0}=-\sin \beta P_{d}^{0}+\cos \beta P_{u}^{0}, \\
& A_{B}^{0}=-\sin \beta_{B} P_{B}^{0}+\cos \beta_{B} \bar{P}_{B}^{0}, \\
& A_{L}^{0}=-\sin \beta_{L} P_{L}^{0}+\cos \beta_{L} \bar{P}_{L}^{0} .
\end{aligned}
$$

At tree level, the masses for those particles are respectively formulated as

$$
\begin{aligned}
m_{A^{0}}^{2} & =\frac{B \mu}{\cos \beta \sin \beta}, \\
m_{A_{B}^{0}}^{2} & =\frac{B_{B} \mu_{B}}{\cos \beta_{B} \sin \beta_{B}}, \\
m_{A_{L}^{0}}^{2} & =\frac{B_{L} \mu_{L}}{\cos \beta_{L} \sin \beta_{L}} .
\end{aligned}
$$

Meanwhile the charged Higgs is

$$
H^{ \pm}=-\sin \beta H_{d}^{ \pm}+\cos \beta H_{u}^{ \pm}
$$

with the tree level mass squared

$$
m_{H^{ \pm}}^{2}=m_{A^{0}}^{2}+m_{\mathrm{W}}^{2}
$$

In the two Higgs doublet sector, the mass squared matrix of neutral CP-even Higgs is diagonalized by the rotation

$$
\left(\begin{array}{l}
H^{0} \\
h^{0}
\end{array}\right)=\left(\begin{array}{cc}
\cos \alpha & \sin \alpha \\
-\sin \alpha & \cos \alpha
\end{array}\right)\left(\begin{array}{c}
H_{d}^{0} \\
H_{u}^{0}
\end{array}\right),
$$

where $h^{0}$ is the lightest neutral CP-even Higgs. 
In the basis $\left(\Phi_{B}^{0}, \varphi_{B}^{0}\right)$, the mass squared matrix is

$$
\mathcal{M}_{E B}^{2}=\left(\begin{array}{ccc}
m_{Z_{B}}^{2} \cos ^{2} \beta_{B}+m_{A_{B}^{0}}^{2} \sin ^{2} \beta_{B}, & \left(m_{Z_{B}}^{2}+m_{A_{B}^{0}}^{2}\right) \cos \beta_{B} \sin \beta_{B} \\
\left(m_{Z_{B}}^{2}+m_{A_{B}^{0}}^{2}\right) \cos \beta_{B} \sin \beta_{B}, & m_{Z_{B}}^{2} \sin ^{2} \beta_{B}+m_{A_{B}^{0}}^{2} \cos ^{2} \beta_{B}
\end{array}\right),
$$

where $m_{Z_{B}}^{2}=g_{B}^{2}\left(v_{B}^{2}+\bar{v}_{B}^{2}\right)$ is mass squared of the neutral $U(1)_{B}$ gauge boson $Z_{B}$. Defining the mixing angle $\alpha_{B}$ through

$$
\tan 2 \alpha_{B}=\frac{m_{z_{B}}^{2}+m_{A_{B}^{0}}^{2}}{m_{Z_{B}}^{2}-m_{A_{B}^{0}}^{2}} \tan 2 \beta_{B}
$$

we obtain two mass eigenstates as

$$
\left(\begin{array}{c}
H_{B}^{0} \\
h_{B}^{0}
\end{array}\right)=\left(\begin{array}{cc}
\cos \alpha_{B} & \sin \alpha_{B} \\
-\sin \alpha_{B} & \cos \alpha_{B}
\end{array}\right)\left(\begin{array}{c}
\Phi_{B}^{0} \\
\varphi_{B}^{0}
\end{array}\right)
$$

Similarly the mass squared matrix for $\left(\Phi_{L}^{0}, \varphi_{L}^{0}\right)$ is written as

$$
\mathcal{M}_{E L}^{2}=\left(\begin{array}{ll}
m_{Z_{L}}^{2} \cos ^{2} \beta_{L}+m_{A_{L}^{0}}^{2} \sin ^{2} \beta_{L}, & \left(m_{z_{L}}^{2}+m_{A_{L}^{0}}^{2}\right) \cos \beta_{L} \sin \beta_{L} \\
\left(m_{Z_{L}}^{2}+m_{A_{L}^{0}}^{2}\right) \cos \beta_{L} \sin \beta_{L}, & m_{z_{L}}^{2} \sin ^{2} \beta_{L}+m_{A_{L}^{0}}^{2} \cos ^{2} \beta_{L}
\end{array}\right),
$$

with $m_{z_{L}}^{2}=4 g_{L}^{2}\left(v_{L}^{2}+\bar{v}_{L}^{2}\right)$ denoting mass squared of the neutral $U(1)_{L}$ gauge boson $Z_{L}$.

In four-component Dirac spinors, the mass matrix for exotic quarks with charged $2 / 3$ is

$$
-\mathcal{L}_{t^{\prime}}^{\text {mass }}=\left(\begin{array}{ll}
\bar{t}_{4 R}^{\prime}, & \bar{t}_{5 R}^{\prime}
\end{array}\right)\left(\begin{array}{ll}
\frac{1}{\sqrt{2}} \lambda_{Q} v_{B}, & -\frac{1}{\sqrt{2}} Y_{u_{5}} v_{d} \\
-\frac{1}{\sqrt{2}} Y_{u_{4}} v_{u}, & \frac{1}{\sqrt{2}} \lambda_{u} \bar{v}_{B}
\end{array}\right)\left(\begin{array}{c}
t_{4 L}^{\prime} \\
t_{5 L}^{\prime}
\end{array}\right)+\text { h.c. }
$$

Using the unitary transformations

$$
\left(\begin{array}{c}
t_{4 L}^{\prime} \\
t_{5 L}^{\prime}
\end{array}\right)=U_{t^{\prime}}^{\dagger} \cdot\left(\begin{array}{c}
t_{4 L} \\
t_{5 L}
\end{array}\right),\left(\begin{array}{c}
t_{4 R}^{\prime} \\
t_{5 R}^{\prime}
\end{array}\right)=W_{t^{\prime}}^{\dagger} \cdot\left(\begin{array}{c}
t_{4 R} \\
t_{5 R}
\end{array}\right),
$$

we diagonalize the mass matrix for the vector quarks with charged $2 / 3$ :

$$
W_{t^{\prime}}^{\dagger} \cdot\left(\begin{array}{ll}
\frac{1}{\sqrt{2}} \lambda_{Q} v_{B}, & -\frac{1}{\sqrt{2}} Y_{u_{5}} v_{d} \\
-\frac{1}{\sqrt{2}} Y_{u_{4}} v_{u}, & \frac{1}{\sqrt{2}} \lambda_{u} \bar{v}_{B}
\end{array}\right) \cdot U_{t^{\prime}}=\operatorname{diag}\left(m_{t_{4}}, m_{t_{5}}\right)
$$

Similarly we can write the mass matrix for the exotic quarks with charged $-1 / 3$ as

$$
-\mathcal{L}_{b^{\prime}}^{\text {mass }}=\left(\begin{array}{ll}
\bar{b}_{4 R}, \bar{b}_{5 R}
\end{array}\right)\left(\begin{array}{l}
-\frac{1}{\sqrt{2}} \lambda_{Q} v_{B},-\frac{1}{\sqrt{2}} Y_{d_{5}} v_{u} \\
-\frac{1}{\sqrt{2}} Y_{d_{4}} v_{d}, \frac{1}{\sqrt{2}} \lambda_{d} \bar{v}_{B}
\end{array}\right)\left(\begin{array}{l}
b_{4 L} \\
b_{5 L}
\end{array}\right)+\text { h.c. }
$$


Adopting the unitary transformations

$$
\left(\begin{array}{c}
b_{4 L}^{\prime} \\
b_{5 L}^{\prime}
\end{array}\right)=U_{b^{\prime}}^{\dagger} \cdot\left(\begin{array}{c}
b_{4 L} \\
b_{5 L}
\end{array}\right),\left(\begin{array}{c}
b_{4 R}^{\prime} \\
b_{5 R}^{\prime}
\end{array}\right)=W_{b^{\prime}}^{\dagger} \cdot\left(\begin{array}{c}
b_{4 R} \\
b_{5 R}
\end{array}\right),
$$

one can diagonalize mass matrix for the vector quarks with charged $-1 / 3$ as

$$
W_{b^{\prime}}^{\dagger} \cdot\left(\begin{array}{c}
-\frac{1}{\sqrt{2}} \lambda_{Q} v_{B},-\frac{1}{\sqrt{2}} Y_{d_{5}} v_{u} \\
-\frac{1}{\sqrt{2}} Y_{d_{4}} v_{d}, \frac{1}{\sqrt{2}} \lambda_{d} \bar{v}_{B}
\end{array}\right) \cdot U_{b^{\prime}}=\operatorname{diag}\left(m_{b_{4}}, m_{b_{5}}\right) .
$$

Assuming CP conservation in exotic quark sector, we then derive the flavor conservative couplings between the lightest neutral CP-even Higgs and charged 2/3 exotic quarks:

$$
\mathcal{L}_{H t^{\prime} t^{\prime}}=\frac{1}{\sqrt{2}} \sum_{i=1}^{2}\left[Y_{u_{4}}\left(W_{t}^{T}\right)_{i 2}\left(U_{t}\right)_{1 i} \cos \alpha+Y_{u_{5}}\left(W_{t}^{T}\right)_{i 1}\left(U_{t}\right)_{2 i} \sin \alpha\right] h^{0} \bar{t}_{i+3} t_{i+3}
$$

where $T$ represents the transposing transformation of a matrix. In a similar way, the flavor conservative couplings between the lightest neutral CP-even Higgs and charged $-1 / 3$ exotic quarks are written as

$$
\mathcal{L}_{H b^{\prime} b^{\prime}}=\frac{1}{\sqrt{2}} \sum_{i=1}^{2}\left[Y_{d_{4}}\left(W_{b}^{T}\right)_{i 2}\left(U_{b}\right)_{1 i} \sin \alpha-Y_{d_{5}}\left(W_{b}^{T}\right)_{i 1}\left(U_{b}\right)_{2 i} \cos \alpha\right] h^{0} \bar{b}_{i+3} b_{i+3} .
$$

Using the superpotential in Eq.(11) and the soft breaking terms, we write the mass squared matrices for exotic scalar quarks as

$$
-\mathcal{L}_{\widetilde{E Q}}^{\text {mass }}=\tilde{t}^{\prime \dagger} \cdot \mathcal{M}_{\tilde{t}^{\prime}}^{2} \cdot \tilde{t}^{\prime}+\tilde{b}^{\prime \dagger} \cdot \mathcal{M}_{\tilde{b}^{\prime}}^{2} \cdot \tilde{b}^{\prime}
$$

with $\tilde{t}^{T}=\left(\tilde{Q}_{4}^{1}, \tilde{U}_{4}^{c *}, \tilde{Q}_{5}^{2 c *}, \tilde{U}_{5}\right), \tilde{b}^{T}=\left(\tilde{Q}_{4}^{2}, \tilde{D}_{4}^{c *}, \tilde{Q}_{5}^{1 c *}, \tilde{D}_{5}^{*}\right)$. The concrete expressions for $4 \times 4$ mass squared matrices $\mathcal{M}_{\tilde{t}^{\prime}}^{2}, \mathcal{M}_{\tilde{b}^{\prime}}^{2}$ are given in appendix $\mathrm{B}$, and the couplings between the lightest neutral CP-even Higgs and exotic scalar quarks are collected in appendix C.

\section{THE LIGHTEST CP-EVEN HIGGS MASS}

It is well known since quite some time that radiative corrections modify the tree level mass squared matrix of neutral Higgs substantially in the MSSM, where the main effect

originates from one-loop diagrams involving the top quark and its scalar partners $\tilde{t}_{1,2}$ [8]. 
In order to obtain masses of the neutral CP-even Higgs reasonably, we should include the radiative corrections from exotic fermions and corresponding supersymmetric partners in the BLMSSM. Then, the mass squared matrix for the neutral CP-even Higgs in the basis $\left(H_{d}^{0}, H_{u}^{0}\right)$ is written as

$$
\mathcal{M}_{\text {even }}^{2}=\left(\begin{array}{cc}
M_{11}^{2}+\Delta_{11} & M_{12}^{2}+\Delta_{12} \\
M_{12}^{2}+\Delta_{12} & M_{22}^{2}+\Delta_{22}
\end{array}\right)
$$

where

$$
\begin{aligned}
& M_{11}^{2}=m_{\mathrm{z}}^{2} \cos ^{2} \beta+m_{A^{0}}^{2} \sin ^{2} \beta, \\
& M_{12}^{2}=-\left(m_{\mathrm{z}}^{2}+m_{A^{0}}^{2}\right) \sin \beta \cos \beta, \\
& M_{22}^{2}=m_{\mathrm{z}}^{2} \sin ^{2} \beta+m_{A^{0}}^{2} \cos ^{2} \beta,
\end{aligned}
$$

and $m_{A^{0}}$ denotes the pseudo-scalar Higgs mass at tree level. The radiative corrections originate from the MSSM sector, exotic fermions and corresponding scalar fermions respectively in this model:

$$
\begin{aligned}
& \Delta_{11}=\Delta_{11}^{M S S M}+\Delta_{11}^{B}+\Delta_{11}^{L}, \\
& \Delta_{12}=\Delta_{12}^{M S S M}+\Delta_{12}^{B}+\Delta_{12}^{L}, \\
& \Delta_{22}=\Delta_{22}^{M S S M}+\Delta_{22}^{B}+\Delta_{22}^{L} .
\end{aligned}
$$

Here the concrete expressions for $\Delta_{11}^{M S S M}, \Delta_{12}^{M S S M}, \Delta_{22}^{M S S M}$ at two-loop level can be found in literature [9], the one-loop radiative corrections from exotic quark fields are formulated as[10]

$$
\begin{aligned}
\Delta_{11}^{B}= & \frac{3 G_{F} Y_{u_{4}}^{4} v^{4}}{4 \sqrt{2} \pi^{2} \sin ^{2} \beta} \cdot \frac{\mu^{2}\left(A_{u_{4}}-\mu \cot \beta\right)^{2}}{\left(m_{\tilde{t}_{1}^{\prime}}^{2}-m_{\tilde{t}_{2}^{\prime}}^{2}\right)^{2}} g\left(m_{\tilde{t}_{1}^{\prime}}, m_{\tilde{t}_{2}^{\prime}}\right) \\
& +\frac{3 G_{F} Y_{u_{5}}^{4} v^{4}}{4 \sqrt{2} \pi^{2} \cos ^{2} \beta}\left\{\ln \frac{m_{\tilde{t}_{3}^{\prime}} m_{\tilde{t}_{4}^{\prime}}}{m_{t_{5}}^{2}}+\frac{A_{u_{5}}\left(A_{u_{5}}-\mu \tan \beta\right)}{m_{\tilde{t}_{3}^{\prime}}^{2}-m_{\tilde{t}_{4}^{\prime}}^{2}} \ln \frac{m_{\tilde{t}_{3}^{\prime}}^{2}}{m_{\tilde{t}_{4}^{\prime}}^{2}}\right. \\
& \left.+\frac{A_{u_{5}}^{2}\left(A_{u_{5}}-\mu \tan \beta\right)^{2}}{\left(m_{\tilde{t}_{3}^{\prime}}^{2}-m_{\tilde{t}_{4}^{\prime}}^{2}\right)^{2}} g\left(m_{\tilde{t}_{3}^{\prime}}, m_{\tilde{t}_{4}^{\prime}}\right)\right\}
\end{aligned}
$$




$$
\begin{aligned}
& +\frac{3 G_{F} Y_{d_{4}}^{4} v^{4}}{4 \sqrt{2} \pi^{2} \cos ^{2} \beta}\left\{\ln \frac{m_{\tilde{b}_{1}^{\prime}} m_{\tilde{b}_{2}^{\prime}}}{m_{b_{4}}^{2}}+\frac{A_{d_{4}}\left(A_{d_{4}}-\mu \tan \beta\right)}{m_{\tilde{b}_{1}^{\prime}}^{2}-m_{\tilde{b}_{2}^{\prime}}^{2}} \ln \frac{m_{\tilde{b}_{1}^{\prime}}^{2}}{m_{\tilde{b}_{2}^{\prime}}^{2}}\right. \\
& \left.+\frac{A_{d_{4}}^{2}\left(A_{d_{4}}-\mu \tan \beta\right)^{2}}{\left(m_{\tilde{b}_{1}^{\prime}}^{2}-m_{\tilde{b}_{2}^{\prime}}^{2}\right)^{2}} g\left(m_{\tilde{b}_{1}^{\prime}}, m_{\tilde{b}_{2}^{\prime}}\right)\right\} \\
& +\frac{3 G_{F} Y_{d_{5}}^{4} v^{4}}{4 \sqrt{2} \pi^{2} \sin ^{2} \beta} \cdot \frac{\mu^{2}\left(A_{d_{5}}-\mu \cot \beta\right)^{2}}{\left(m_{\tilde{b}_{3}^{\prime}}^{2}-m_{\tilde{b}_{4}^{\prime}}^{2}\right)^{2}} g\left(m_{\tilde{b}_{3}^{\prime}}, m_{\tilde{b}_{4}^{\prime}}\right) \text {, } \\
& \Delta_{12}^{B}=\frac{3 G_{F} Y_{u_{4}}^{4} v^{4}}{8 \sqrt{2} \pi^{2} \sin ^{2} \beta} \cdot \frac{\mu\left(-A_{u_{4}}+\mu \cot \beta\right)}{m_{\tilde{t}_{1}^{\prime}}^{2}-m_{\tilde{t}_{2}^{\prime}}^{2}}\left\{\ln \frac{m_{\tilde{t}_{1}^{\prime}}}{m_{\tilde{t}_{2}^{\prime}}}+\frac{A_{u_{4}}\left(A_{u_{4}}-\mu \cot \beta\right)}{m_{\tilde{t}_{1}^{\prime}}^{2}-m_{\tilde{t}_{2}^{\prime}}^{2}} g\left(m_{\tilde{t}_{1}^{\prime}}, m_{\tilde{t}_{2}^{\prime}}\right)\right\} \\
& +\frac{3 G_{F} Y_{u_{5}}^{4} v^{4}}{8 \sqrt{2} \pi^{2} \cos ^{2} \beta} \cdot \frac{\mu\left(-A_{u_{5}}+\mu \tan \beta\right)}{m_{\tilde{t}_{3}^{\prime}}^{2}-m_{\tilde{t}_{4}^{\prime}}^{2}}\left\{\ln \frac{m_{\tilde{t}_{3}^{\prime}}}{m_{\tilde{t}_{4}^{\prime}}}+\frac{A_{u_{5}}\left(A_{u_{5}}-\mu \tan \beta\right)}{m_{\tilde{t}_{3}^{\prime}}^{2}-m_{\tilde{t}_{4}^{\prime}}^{2}} g\left(m_{\tilde{t}_{3}^{\prime}}, m_{\tilde{t}_{4}^{\prime}}\right)\right\} \\
& +\frac{3 G_{F} Y_{d_{4}}^{4} v^{4}}{8 \sqrt{2} \pi^{2} \cos ^{2} \beta} \cdot \frac{\mu\left(-A_{d_{4}}+\mu \tan \beta\right)}{m_{\tilde{d}_{1}^{\prime}}^{2}-m_{\tilde{d}_{2}^{\prime}}^{2}}\left\{\ln \frac{m_{\tilde{d}_{1}^{\prime}}}{m_{\tilde{d}_{2}^{\prime}}}+\frac{A_{d_{4}}\left(A_{d_{4}}-\mu \tan \beta\right)}{m_{\tilde{d}_{1}^{\prime}}^{2}-m_{\tilde{d}_{2}^{\prime}}^{2}} g\left(m_{\tilde{d}_{1}^{\prime}}, m_{\tilde{d}_{2}^{\prime}}\right)\right\} \\
& +\frac{3 G_{F} Y_{d_{5}}^{4} v^{4}}{8 \sqrt{2} \pi^{2} \sin ^{2} \beta} \cdot \frac{\mu\left(-A_{d_{5}}+\mu \cot \beta\right)}{m_{\tilde{b}_{3}^{\prime}}^{2}-m_{\tilde{b}_{4}^{\prime}}^{2}}\left\{\ln \frac{m_{\tilde{b}_{3}^{\prime}}}{m_{\tilde{b}_{4}^{\prime}}}+\frac{A_{d_{5}}\left(A_{d_{5}}-\mu \cot \beta\right)}{m_{\tilde{b}_{3}^{\prime}}^{2}-m_{\tilde{b}_{4}^{\prime}}^{2}} g\left(m_{\tilde{b}_{3}^{\prime}}, m_{\tilde{b}_{4}^{\prime}}\right)\right\}, \\
& \Delta_{22}^{B}=\frac{3 G_{F} Y_{u_{4}}^{4} v^{4}}{4 \sqrt{2} \pi^{2} \sin ^{2} \beta}\left\{\ln \frac{m_{\tilde{t}_{1}^{\prime}} m_{\tilde{t}_{2}^{\prime}}}{m_{t_{4}}^{2}}+\frac{A_{u_{4}}\left(A_{u_{4}}-\mu \cot \beta\right)}{m_{\tilde{t}_{1}^{\prime}}^{2}-m_{\tilde{t}_{2}^{\prime}}^{2}} \ln \frac{m_{\tilde{t}_{1}^{\prime}}^{2}}{m_{\tilde{t}_{2}^{\prime}}^{2}}\right. \\
& \left.+\frac{A_{u_{4}}^{2}\left(A_{u_{4}}-\mu \cot \beta\right)^{2}}{\left(m_{\tilde{t}_{1}^{\prime}}^{2}-m_{\tilde{t}_{2}^{\prime}}^{2}\right)^{2}} g\left(m_{\tilde{t}_{1}^{\prime}}, m_{\tilde{t}_{2}^{\prime}}\right)\right\} \\
& +\frac{3 G_{F} Y_{u_{5}}^{4} v^{4}}{4 \sqrt{2} \pi^{2} \cos ^{2} \beta} \cdot \frac{\mu^{2}\left(A_{u_{5}}-\mu \tan \beta\right)^{2}}{\left(m_{\tilde{t}_{3}^{\prime}}^{2}-m_{\tilde{t}_{4}^{\prime}}^{2}\right)^{2}} g\left(m_{\tilde{t}_{3}^{\prime}}, m_{\tilde{t}_{4}^{\prime}}\right) \\
& +\frac{3 G_{F} Y_{d_{4}}^{4} v^{4}}{4 \sqrt{2} \pi^{2} \cos ^{2} \beta} \cdot \frac{\mu^{2}\left(A_{d_{4}}-\mu \tan \beta\right)^{2}}{\left(m_{\tilde{b}_{1}^{\prime}}^{2}-m_{\tilde{b}_{2}^{\prime}}^{2}\right)^{2}} g\left(m_{\tilde{b}_{1}^{\prime}}, m_{\tilde{b}_{2}^{\prime}}\right) \\
& +\frac{3 G_{F} Y_{d_{5}}^{4} v^{4}}{4 \sqrt{2} \pi^{2} \sin ^{2} \beta}\left\{\ln \frac{m_{\tilde{b}_{3}^{\prime}} m_{\tilde{b}_{4}^{\prime}}}{m_{b_{5}}^{2}}+\frac{A_{d_{5}}\left(A_{d_{5}}-\mu \cot \beta\right)}{m_{\tilde{b}_{3}^{\prime}}^{2}-m_{\tilde{b}_{4}^{\prime}}^{2}} \ln \frac{m_{\tilde{b}_{3}^{\prime}}^{2}}{m_{\tilde{b}_{4}^{\prime}}^{2}}\right. \\
& \left.+\frac{A_{d_{5}}^{2}\left(A_{d_{5}}-\mu \cot \beta\right)^{2}}{\left(m_{\tilde{b}_{3}^{\prime}}^{2}-m_{\tilde{b}_{4}^{\prime}}^{2}\right)^{2}} g\left(m_{\tilde{b}_{3}^{\prime}}, m_{\tilde{b}_{4}^{\prime}}\right)\right\} \text {, }
\end{aligned}
$$

here $v=\sqrt{v_{u}^{2}+v_{d}^{2}} \simeq 246 \mathrm{GeV}$ and

$$
g(x, y)=1-\frac{x^{2}+y^{2}}{x^{2}-y^{2}} \ln \frac{x}{y}
$$


To derive the results presented in Eq.(28), we adopt the assumption $\left|\lambda_{Q} v_{B}\right|, \quad\left|\lambda_{u} \bar{v}_{B}\right|, \quad\left|\lambda_{d} \bar{v}_{B}\right| \gg\left|Y_{u_{4}} v\right|,\left|Y_{u_{5}} v\right|,\left|Y_{d_{4}} v\right|, \quad\left|Y_{d_{5}} v\right|$ in our calculation. Similarly, one can obtain the one-loop radiative corrections from exotic lepton fields presented in appendix D.

One most stringent constraint on parameter space of the BLMSSM is that the mass squared matrix in Eq.(25) should produce an eigenvalue around $(125 \mathrm{GeV})^{2}$ as mass squared of the lightest neutral CP-even Higgs. The current combination of the ATLAS and CMS data gives[1, 2, 11]:

$$
m_{h^{0}}=125.9 \pm 2.1 \mathrm{GeV}
$$

this fact constrains parameter space of the BLMSSM strongly.

IV. $g g \rightarrow h^{0}$ AND $h^{0} \rightarrow \gamma \gamma, Z Z^{*}, W W^{*}$

The Higgs is produced chiefly through the gluon fusion at the LHC. In the SM, the leading order (LO) contributions originate from the one loop diagram which involves virtual top quarks. The cross section for this process is known to the next-to-next-to-leading order (NNLO) [12] which can enhance the LO result by 80-100\%. Furthermore, any new particle which strongly couples with the Higgs can significantly modified this cross section. In supersymmetric extension of the SM considered here, the LO decay width for the process $h^{0} \rightarrow g g$ is given by (see Ref.[13] and references therein)

$$
\Gamma_{N P}\left(h^{0} \rightarrow g g\right)=\frac{G_{F} \alpha_{s}^{2} m_{h^{0}}^{3}}{64 \sqrt{2} \pi^{3}}\left|\sum_{q} g_{h^{0} q q} A_{1 / 2}\left(x_{q}\right)+\sum_{\tilde{q}} g_{h^{0} \tilde{q} \tilde{q}} \frac{m_{\mathrm{z}}^{2}}{m_{\tilde{q}}^{2}} A_{0}\left(x_{\tilde{q}}\right)\right|^{2}
$$

with $x_{a}=m_{h^{0}}^{2} /\left(4 m_{a}^{2}\right)$. In addition, $q=t, b, t_{4}, t_{5}, b_{4}, b_{5}$ and $\tilde{q}=\tilde{t}_{1,2}, \tilde{b}_{1,2}, \tilde{\mathcal{U}}_{i}, \tilde{\mathcal{D}}_{i}(i=$ $1,2,3,4)$. The concrete expressions for $g_{h^{0} t t}, g_{h^{0} b b}, g_{h^{0} \tilde{t}_{i} \tilde{t}_{i}}, g_{h^{0} \tilde{b}_{i} \tilde{b}_{i}},(i=1,2)$ can be found in literature[6], and

$$
\begin{aligned}
g_{h^{0} t_{4} t_{4}} & =-\frac{\sqrt{2} m_{\mathrm{W}} s_{\mathrm{W}}}{e m_{t_{4}}}\left[Y_{u_{4}}\left(W_{t}^{T}\right)_{12}\left(U_{t}\right)_{11} \cos \alpha+Y_{u_{5}}\left(W_{t}^{T}\right)_{11}\left(U_{t}\right)_{21} \sin \alpha\right], \\
g_{h^{0} t_{5} t_{5}} & =-\frac{\sqrt{2} m_{\mathrm{W}} s_{\mathrm{W}}}{e m_{t_{5}}}\left[Y_{u_{4}}\left(W_{t}^{T}\right)_{22}\left(U_{t}\right)_{12} \cos \alpha+Y_{u_{5}}\left(W_{t}^{T}\right)_{21}\left(U_{t}\right)_{22} \sin \alpha\right],
\end{aligned}
$$




$$
\begin{aligned}
g_{h^{0} b_{4} b_{4}} & =-\frac{\sqrt{2} m_{\mathrm{W}} s_{\mathrm{W}}}{e m_{b_{4}}}\left[Y_{d_{4}}\left(W_{b}^{T}\right)_{12}\left(U_{b}\right)_{11} \sin \alpha-Y_{d_{5}}\left(W_{b}^{T}\right)_{11}\left(U_{b}\right)_{21} \cos \alpha\right], \\
g_{h^{0} b_{5} b_{5}} & =-\frac{\sqrt{2} m_{\mathrm{W}} s_{\mathrm{W}}}{e m_{b_{5}}}\left[Y_{d_{4}}\left(W_{b}^{T}\right)_{22}\left(U_{b}\right)_{12} \sin \alpha-Y_{d_{5}}\left(W_{b}^{T}\right)_{21}\left(U_{b}\right)_{22} \cos \alpha\right], \\
g_{h^{0} \tilde{\mathcal{U}}_{i} \tilde{\mathcal{u}}_{i}} & =-\frac{m_{\mathrm{W}}^{2} s_{\mathrm{W}}}{e m_{\tilde{\mathcal{u}}_{i}}^{2}}\left[\xi_{u i i}^{S} \cos \alpha-\xi_{d i i}^{S} \sin \alpha\right], \quad(i=1,2,3,4), \\
g_{h^{0} \tilde{\mathcal{D}}_{i} \tilde{\mathcal{D}}_{i}} & =-\frac{m_{\mathrm{W}}^{2} s_{\mathrm{W}}}{e m_{\tilde{\mathcal{D}}_{i}}^{2}}\left[\eta_{u i i}^{S} \cos \alpha-\eta_{d i i}^{S} \sin \alpha\right], \quad(i=1,2,3,4) .
\end{aligned}
$$

Here, we adopt the abbreviation $s_{\mathrm{w}}=\sin \theta_{\mathrm{w}}$ with $\theta_{\mathrm{w}}$ denoting the Weinberg angle. Furthermore, $e$ is the electromagnetic coupling constant, and the concrete expressions of $\xi_{u i i}^{S}, \xi_{d i i}^{S}, \eta_{u i i}^{S}, \eta_{d i i}^{S}$ can be found in appendix C. The form factors $A_{1 / 2}, A_{0}$ in Eq. (31) are defined as

$$
\begin{aligned}
& A_{1 / 2}(x)=2[x+(x-1) g(x)] / x^{2}, \\
& A_{0}(x)=-(x-g(x)) / x^{2},
\end{aligned}
$$

with

$$
g(x)=\left\{\begin{array}{l}
\arcsin ^{2} \sqrt{x}, x \leq 1 \\
-\frac{1}{4}\left[\ln \frac{1+\sqrt{1-1 / x}}{1-\sqrt{1-1 / x}}-i \pi\right]^{2}, x>1
\end{array}\right.
$$

The Higgs to diphoton decay is also obtained from loop diagrams, the LO contributions are derived from the one loop diagrams containing virtual charged gauge boson $W^{ \pm}$or virtual top quarks in the SM. In the BLMSSM, the exotic fermions $t_{4,5}, b_{4,5}, e_{4,5}$ together with their supersymmetric partners contribute the corrections to the decay width of Higgs to diphoton at LO, the corresponding expression is written as

$$
\begin{aligned}
\Gamma_{N P}\left(h^{0} \rightarrow \gamma \gamma\right)= & \frac{G_{F} \alpha^{2} m_{h^{0}}^{3}}{128 \sqrt{2} \pi^{3}} \mid \sum_{f} N_{c} Q_{f}^{2} g_{h^{0} f f} A_{1 / 2}\left(x_{f}\right)+g_{h^{0} W W} A_{1}\left(x_{\mathrm{W}}\right) \\
& +g_{h^{0} H^{+} H^{-}} \frac{m_{\mathrm{W}}^{2}}{m_{H^{ \pm}}^{2}} A_{0}\left(x_{H^{ \pm}}\right)+\sum_{i=1}^{2} g_{h^{0} \chi_{i}^{+} \chi_{i}^{-}} \frac{m_{\mathrm{W}}}{m_{\chi_{i}}} A_{1 / 2}\left(x_{\chi_{i}}\right) \\
& +\left.\sum_{\tilde{f}} N_{c} Q_{f}^{2} g_{h^{0} \tilde{f} \tilde{f}} \frac{m_{\mathrm{z}}^{2}}{m_{\tilde{f}}^{2}} A_{0}\left(x_{\tilde{f}}\right)\right|^{2},
\end{aligned}
$$


where $g_{h^{0} W W}=\sin (\beta-\alpha)$, the concrete expression for the loop functions $A_{1}$ is

$$
A_{1}(x)=-\left[2 x^{2}+3 x+3(2 x-1) g(x)\right] / x^{2} .
$$

The concrete expressions for $g_{h^{0} x_{i}^{+} x_{i}^{-}}, g_{h^{0} H^{+} H^{-}}$and the couplings between the lightest neutral CP-even Higgs and exotic leptons/sleptons can also be found in literature [6].

For the lightest neutral CP-even Higgs around $125 \mathrm{GeV}$ mass, it can decay through the modes $h^{0} \rightarrow Z Z^{*}, h^{0} \rightarrow W W^{*}$ where $Z^{*} / W^{*}$ denotes the off-shell neutral/charged electroweak gauge bosons. Summing over all channels available to the $W^{*}$ or $Z^{*}$, one can write the widths as $[14,15]$

$$
\begin{aligned}
& \Gamma\left(h^{0} \rightarrow W W^{*}\right)=\frac{3 e^{4} m_{h^{0}}}{512 \pi^{3} s_{\mathrm{W}}^{4}}\left|g_{h^{0} W W}\right|^{2} F\left(\frac{m_{\mathrm{W}}}{m_{h^{0}}}\right), \\
& \Gamma\left(h^{0} \rightarrow Z Z^{*}\right)=\frac{e^{4} m_{h^{0}}}{2048 \pi^{3} s_{\mathrm{w}}^{4} c_{\mathrm{w}}^{4}}\left|g_{h^{0} Z Z}\right|^{2}\left(7-\frac{40}{3} s_{\mathrm{w}}^{2}+\frac{160}{9} s_{\mathrm{w}}^{4}\right) F\left(\frac{m_{\mathrm{z}}}{m_{h^{0}}}\right),
\end{aligned}
$$

with $g_{h^{0} Z Z}=g_{h^{0} W W}$ and the abbreviation $c_{\mathrm{w}}=\cos \theta_{\mathrm{w}}$. The form factor $F(x)$ is given as

$$
\begin{aligned}
F(x)= & -\left(1-x^{2}\right)\left(\frac{47}{2} x^{2}-\frac{13}{2}+\frac{1}{x^{2}}\right)-3\left(1-6 x^{2}+4 x^{4}\right) \ln x \\
& +\frac{3\left(1-8 x^{2}+20 x^{4}\right)}{\sqrt{4 x^{2}-1}} \cos ^{-1}\left(\frac{3 x^{2}-1}{2 x^{3}}\right) .
\end{aligned}
$$

Besides the Higgs discovery the ATLAS and CMS experiments have both observed an excess in Higgs production and decay into diphoton channel which is a factor $1.4 \sim 2$ times larger than the SM expectations. The observed signals for the diphoton and $Z Z^{*}, W W^{*}$ channels are quantified by the ratios

$$
\begin{aligned}
& R_{\gamma \gamma}=\frac{\Gamma_{N P}\left(h_{0} \rightarrow g g\right) \Gamma_{N P}\left(h_{0} \rightarrow \gamma \gamma\right)}{\Gamma_{S M}\left(h_{0} \rightarrow g g\right) \Gamma_{S M}\left(h_{0} \rightarrow \gamma \gamma\right)}, \\
& R_{V V^{*}}=\frac{\Gamma_{N P}\left(h_{0} \rightarrow g g\right) \Gamma_{N P}\left(h_{0} \rightarrow V V^{*}\right)}{\Gamma_{S M}\left(h_{0} \rightarrow g g\right) \Gamma_{S M}\left(h_{0} \rightarrow V V^{*}\right)}, \quad(V=Z, W) .
\end{aligned}
$$


The current values of the ratios are $[1,2,11]$ :

$$
\begin{array}{ll}
\text { ATLAS + CMS }: & R_{\gamma \gamma}=1.77 \pm 0.33, \\
\text { ATLAS + CMS : } & R_{V V^{*}}=0.94 \pm 0.40,(V=Z, W) .
\end{array}
$$

Note that the combinations of the ATLAS and CMS results are taken from Ref.[11].

\section{NUMERICAL ANALYSES}

As mentioned above, the most stringent constraint on the parameter space is that the $2 \times 2$ mass squared matrix in Eq.(25) should predict the lightest eigenvector with a mass $m_{h_{0}} \simeq 125.9 \mathrm{GeV}$. In order to obtain the final results coinciding with this condition, we require the tree level mass of CP-odd Higgs $m_{A^{0}}$ satisfying

$$
m_{A^{0}}^{2}=\frac{m_{h_{0}}^{2}\left(m_{\mathrm{z}}^{2}-m_{h_{0}}^{2}+\Delta_{11}+\Delta_{22}\right)-m_{\mathrm{z}}^{2} \Delta_{A}+\Delta_{12}^{2}-\Delta_{11} \Delta_{22}}{-m_{h_{0}}^{2}+m_{\mathrm{z}}^{2} \cos ^{2} 2 \beta+\Delta_{B}},
$$

where

$$
\begin{aligned}
& \Delta_{A}=\sin ^{2} \beta \Delta_{11}+\cos ^{2} \beta \Delta_{22}+\sin 2 \beta \Delta_{12}, \\
& \Delta_{B}=\cos ^{2} \beta \Delta_{11}+\sin ^{2} \beta \Delta_{22}+\sin 2 \beta \Delta_{12} .
\end{aligned}
$$

Through scanning the parameter space, we find the evaluations on $R_{\gamma \gamma}, R_{V V^{*}}$ and masses of the heaviest CP-even Higgs and CP-odd Higgs depending on $\tan \beta$ acutely as $m_{h^{0}}=$ $125.9 \mathrm{GeV}$. In our numerical analysis, we adopt the ansatz on relevant parameter space

$$
\begin{aligned}
& B_{4}=L_{4}=\frac{3}{2}, \\
& m_{\tilde{Q}_{3}}=m_{\tilde{U}_{3}}=m_{\tilde{D}_{3}}=1 \mathrm{TeV}, \\
& m_{\tilde{U}_{4}}=m_{\tilde{D}_{4}}=m_{\tilde{Q}_{5}}=m_{\tilde{U}_{5}}=m_{\tilde{D}_{5}}=1 \mathrm{TeV}, \\
& m_{\tilde{L}_{4}}=m_{\tilde{\nu}_{4}}=m_{\tilde{E}_{4}}=m_{\tilde{L}_{5}}=m_{\tilde{\nu}_{5}}=m_{\tilde{E}_{5}}=1 \mathrm{TeV}, \\
& m_{Z_{B}}=m_{z_{L}}=1 \mathrm{TeV},
\end{aligned}
$$



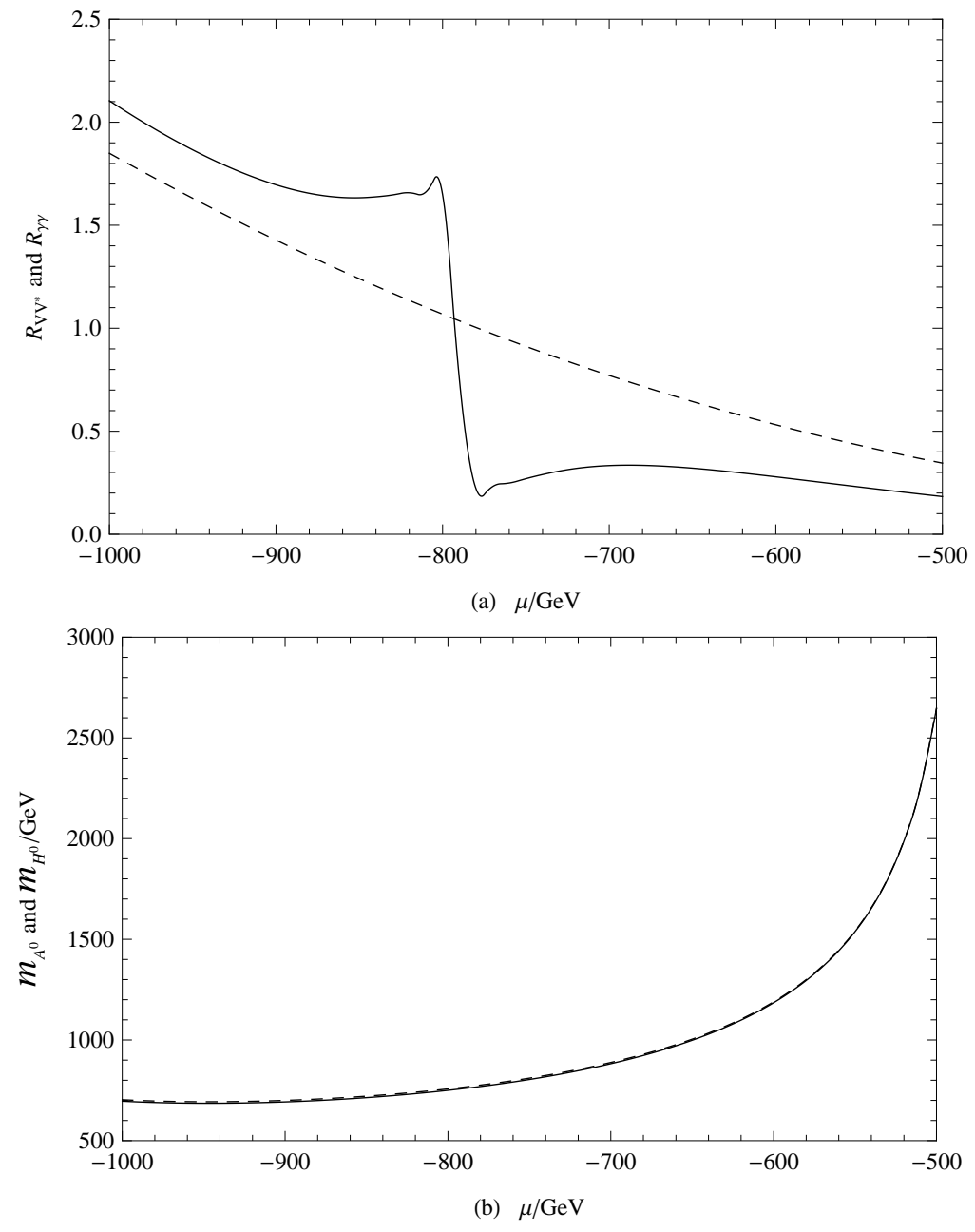

FIG. 1: As $Y_{u_{5}}=0.7 Y_{b}, Y_{d_{5}}=0.13 Y_{t}, m_{\tilde{Q}_{4}}=790 \mathrm{GeV}$ and $A_{t}=-1 \mathrm{TeV}$, (a) $R_{\gamma \gamma}$ (solid-line) and $R_{V V^{*}}$ (dashed-line) vary with the parameter $\mu$, and (b) $m_{A^{0}}$ (solid-line) and $m_{H^{0}}$ (dashed-line) vary with the parameter $\mu$, respectively.

$$
\begin{aligned}
& A_{\nu_{4}}=A_{e_{4}}=A_{\nu_{5}}=A_{e_{4}}=A_{d_{4}}=A_{u_{5}}=A_{d_{5}}=550 \mathrm{GeV}, \\
& v_{B_{t}}=\sqrt{v_{B}^{2}+\bar{v}_{B}^{2}}=3 \mathrm{TeV}, \quad v_{L_{t}}=\sqrt{v_{L}^{2}+\bar{v}_{L}^{2}}=3 \mathrm{TeV}, \\
& A_{B Q}=A_{B U}=A_{B D}=-A_{b}=1 \mathrm{TeV}, \\
& Y_{u_{4}}=0.76 Y_{t}, \quad Y_{d_{4}}=0.7 Y_{b}, \quad \lambda_{Q}=\lambda_{u}=\lambda_{d}=0.5 \\
& m_{2}=750 \mathrm{GeV}, \quad \mu_{B}=500 \mathrm{GeV}, \\
& \tan \beta=\tan \beta_{B}=\tan \beta_{L}=2,
\end{aligned}
$$




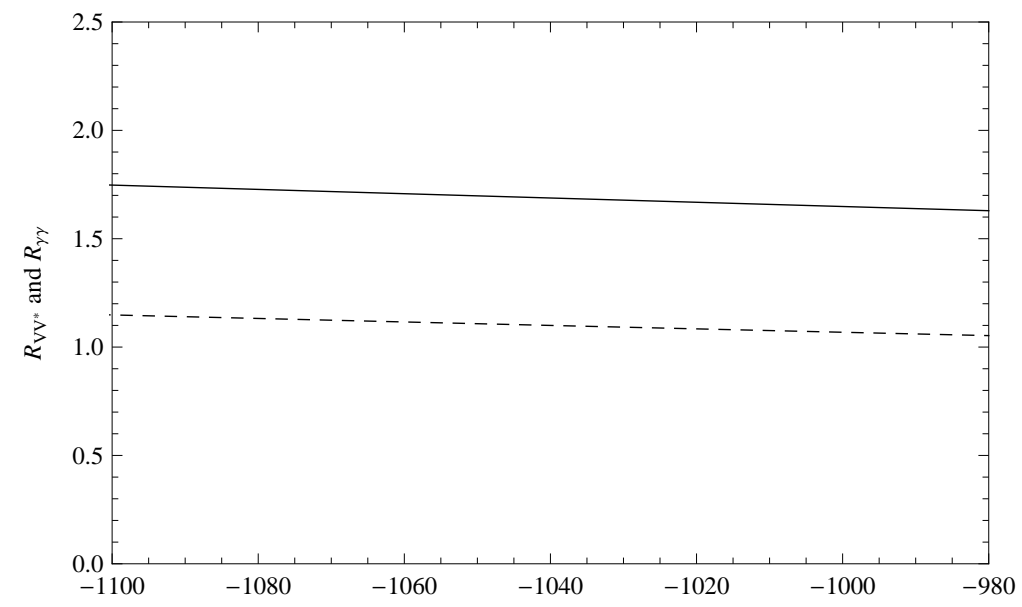

(a) $A_{t} / \mathrm{GeV}$

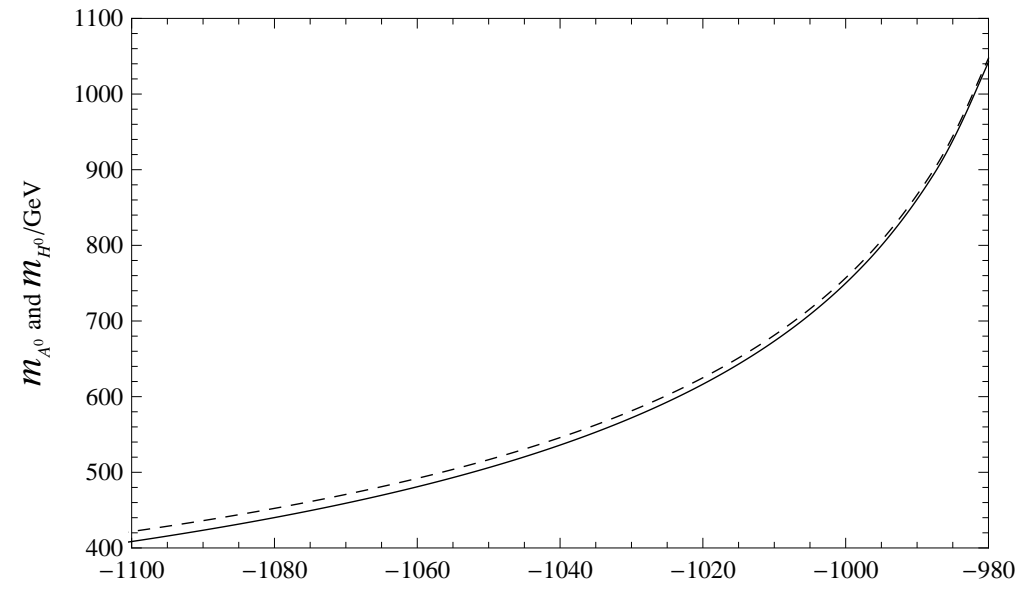

(b) $A_{t} / \mathrm{GeV}$

FIG. 2: As $Y_{u_{5}}=0.7 Y_{b}, Y_{d_{5}}=0.13 Y_{t}, m_{\tilde{Q}_{4}}=790 \mathrm{GeV}$ and $\mu=-800 \mathrm{GeV}$, (a) $R_{\gamma \gamma}$ (solid-line) and $R_{V V^{*}}$ (dashed-line) vary with the parameter $A_{t}$, and (b) $m_{A^{0}}$ (solid-line) and $m_{H^{0}}$ (dashed-line) vary with the parameter $A_{t}$, respectively.

to reduce the number of free parameters in the model considered here. Furthermore, we choose the masses for exotic leptons from Ref.[6]:

$$
m_{\nu_{4}}=m_{\nu_{5}}=90 \mathrm{GeV}, m_{e_{4}}=m_{e_{5}}=100 \mathrm{GeV} .
$$

For relevant parameters in the SM, we choose 16]

$$
\alpha_{s}\left(m_{\mathrm{z}}\right)=0.118, \quad \alpha\left(m_{\mathrm{z}}\right)=1 / 128, \quad s_{\mathrm{W}}^{2}\left(m_{\mathrm{z}}\right)=0.23,
$$



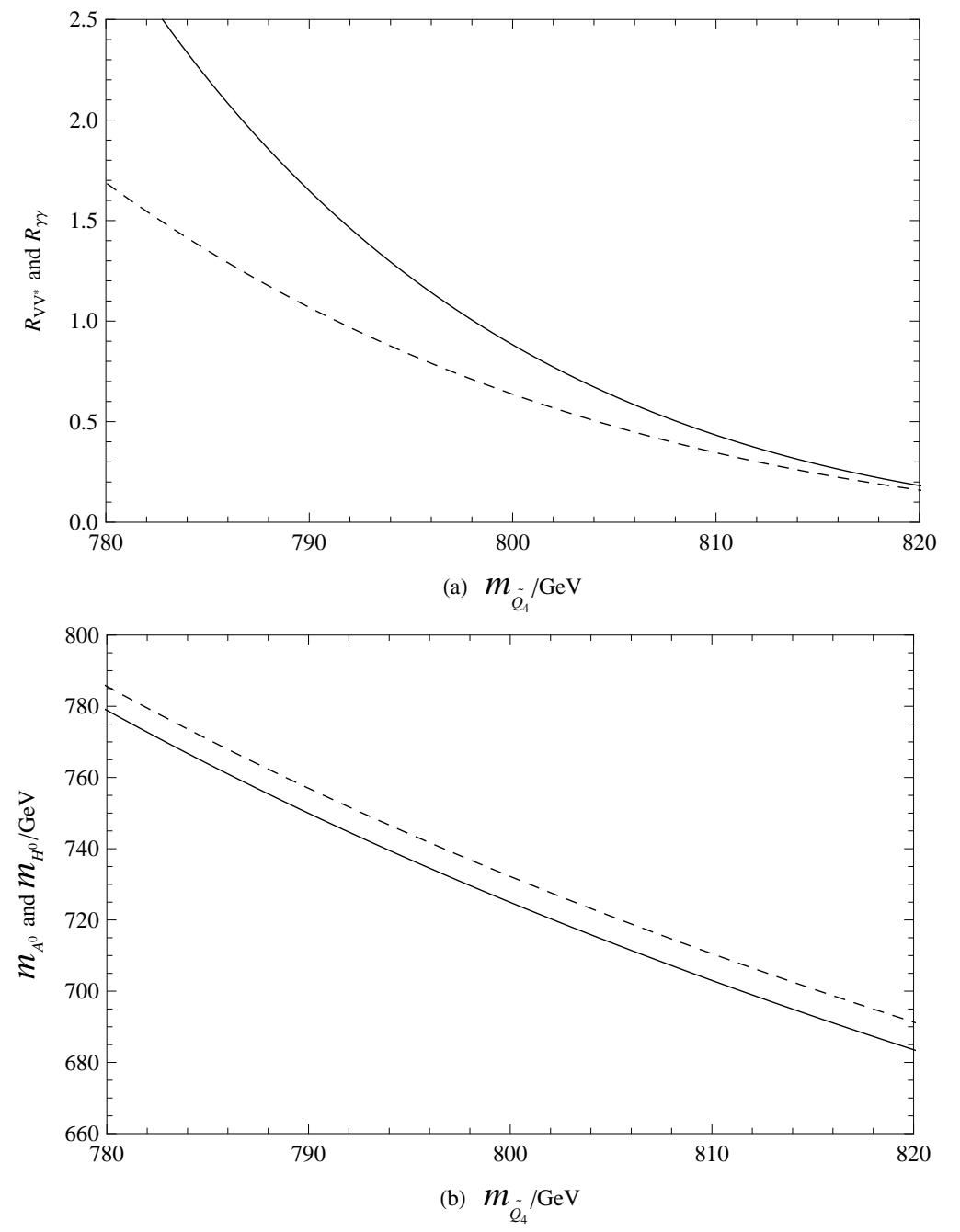

FIG. 3: As $Y_{u_{5}}=0.7 Y_{b}, Y_{d_{5}}=0.13 Y_{t}, A_{t}=-1 \mathrm{TeV}$ and $\mu=-800 \mathrm{GeV}$, (a) $R_{\gamma \gamma}$ (solid-line) and $R_{V V^{*}}$ (dashed-line) vary with the parameter $m_{\tilde{Q}_{4}}$, and (b) $m_{A^{0}}$ (solid-line) and $m_{H^{0}}$ (dashed-line) vary with the parameter $m_{\tilde{Q}_{4}}$, respectively.

$$
m_{t}=174.2 \mathrm{GeV}, m_{b}=4.2 \mathrm{GeV}, m_{\mathrm{w}}=80.4 \mathrm{GeV} .
$$

Considering that the CMS collaboration has excluded a SM Higgs with mass in the range 127.5 GeV - $600 \mathrm{GeV}$, we require the theoretical evaluations on masses of the heaviest CPeven Higgs and CP-odd Higgs respectively in the range $m_{A^{0}} \geq 600 \mathrm{GeV}, m_{H^{0}} \geq 600 \mathrm{GeV}$. Choosing $Y_{u_{5}}=Y_{d_{4}}=0.7 Y_{b}, Y_{d_{5}}=0.13 Y_{t}, m_{\tilde{Q}_{4}}=790 \mathrm{GeV}$ and $A_{t}=-1 \mathrm{TeV}$, we plot $R_{\gamma \gamma}$ (solid-line) and $R_{V V^{*}}$ (dashed-line) varying with the parameter $\mu$ in Fig.1.(a), and plot $m_{A^{0}}$ 


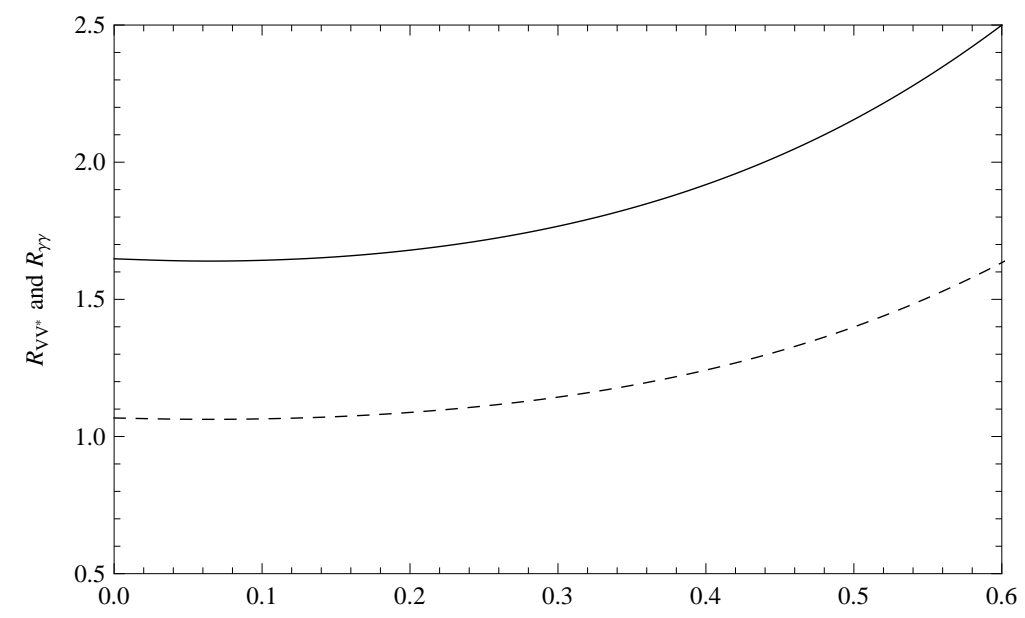

(a) $Y_{\mathrm{d} 5} / Y_{t}$

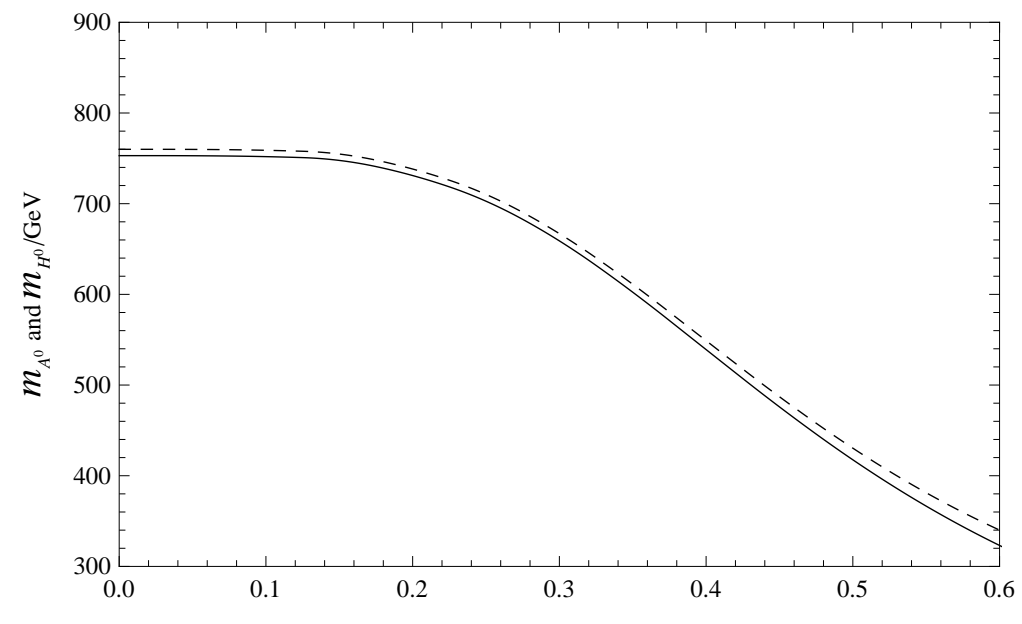

(b) $Y_{\mathrm{d} 5} / Y_{t}$

FIG. 4: As $Y_{u_{5}}=0.7 Y_{b}, A_{t}=-1 \mathrm{TeV}$ and $\mu=-790 \mathrm{GeV}$, (a) $R_{\gamma \gamma}$ (solid-line) and $R_{V V^{*}}$ (dashedline) vary with the ratio $Y_{d_{5}} / Y_{t}$, and (b) $m_{A^{0}}$ (solid-line) and $m_{H^{0}}$ (dashed-line) vary with the ratio $Y_{d_{5}} / Y_{t}$, respectively.

(solid-line) and $m_{H^{0}}$ (dashed-line) varying with the parameter $\mu$ in Fig 1 (b), respectively. Using our assumptions on relevant parameter space in the BLMSSM, we find the theoretical evaluations on $R_{\gamma \gamma}, R_{V V^{*}}, m_{A^{0}}$ and $m_{H^{0}}$ depending on $\mu$ acutely. As $-900 \mathrm{GeV} \leq \mu \leq$ $-800 \mathrm{GeV}$, the theoretical predictions on $R_{\gamma \gamma}$ and $R_{V V^{*}}$ are all coincide with experimental data in Eq.(40), and masses of the heaviest CP-even Higgs and CP-odd Higgs $m_{A^{0}} \sim m_{H^{0}} \geq$ $700 \mathrm{GeV}$ simultaneously. 
Another parameter $A_{t}$ maybe affects the theoretical evaluations on $R_{\gamma \gamma}$ and $R_{V V^{*}}$ strongly here. Taking $Y_{u_{5}}=Y_{d_{4}}=0.7 Y_{b}, Y_{d_{5}}=0.13 Y_{t}, m_{\tilde{Q}_{4}}=790 \mathrm{GeV}$ and $\mu=-800 \mathrm{GeV}$, we depict $R_{\gamma \gamma}$ (solid-line) and $R_{V V^{*}}$ (dashed-line) varying with the parameter $A_{t}$ in Fig[2(a), and plot $m_{A^{0}}$ (solid-line) and $m_{H^{0}}$ (dashed-line) varying with the parameter $A_{t}$ in Fig 2(b), respectively. Under our assumptions on the parameter space, the dependence of $R_{\gamma \gamma}$ and $R_{V V^{*}}$ on $A_{t}$ is very mild. Nevertheless, the theoretical evaluations of $m_{A^{0}}$ and $m_{H^{0}}$ depend on $A_{t}$ strongly. When $A_{t} \geq-1 \mathrm{TeV}$, the theoretical evaluations on $R_{\gamma \gamma}$ and $R_{V V^{*}}$ are all coincide with experimental data in Eq.(40), and masses of the heaviest CP-even Higgs and CP-odd Higgs $m_{A^{0}} \sim m_{H^{0}} \geq 700 \mathrm{GeV}$ meantime.

Besides those parameters existing in the MSSM already, the 'brand new' parameters in the BLMSSM also affect the theoretical evaluations on $R_{\gamma \gamma}, R_{V V^{*}}$ and $m_{A^{0}}, m_{H^{0}}$ strongly as $m_{h^{0}}=125.9 \mathrm{GeV}$. In Fig.(3) , we investigate $R_{\gamma \gamma}, R_{V V^{*}}$ and $m_{A^{0}}, m_{H^{0}}$ versus the soft mass of fourth generation left-handed scalar quarks $m_{\tilde{Q}_{4}}$. Where the solid line in Fig.(3) (a) represents $R_{\gamma \gamma}$ varying with $m_{\tilde{Q}_{4}}$, the dashed line in Fig.(3)(a) represents $R_{V V^{*}}$ varying with $m_{\tilde{Q}_{4}}$, the solid line in Fig.(3) (b) represents $m_{A^{0}}$ varying with $m_{\tilde{Q}_{4}}$, the dashed line in Fig.(3) (b) represents $m_{H^{0}}$ varying with $m_{\tilde{Q}_{4}}$, respectively. Actually, the theoretical evaluations on $R_{\gamma \gamma}$, $R_{V V^{*}}, m_{A^{0}}$ and $m_{H^{0}}$ decrease steeply with the increasing of $m_{\tilde{Q}_{4}}$. When $m_{\tilde{Q}_{4}} \geq 800 \mathrm{GeV}$, the theoretical prediction on $R_{\gamma \gamma}$ already lies out the experimental range in Eq.(40). In Fig.(44), we investigate the theoretical predictions on $R_{\gamma \gamma}, R_{V V^{*}}$ and $m_{A^{0}}, m_{H^{0}}$ versus the Yukawa coupling of fifth generation down-type quark $Y_{d_{5}}$. Where the solid line in Fig.(4) (a) represents $R_{\gamma \gamma}$ varying with $Y_{d_{5}}$, the dashed line in Fig.(4)(a) represents $R_{V V^{*}}$ varying with $Y_{d_{5}}$, the solid line in Fig.(44)(b) represents $m_{A^{0}}$ varying with $Y_{d_{5}}$, the dashed line in Fig.(44)(b) represents $m_{H^{0}}$ varying with $Y_{d_{5}}$, respectively. In fact, the theoretical evaluations on $R_{\gamma \gamma}$ and $R_{V V^{*}}$ raise slowly with increasing of the ratio $Y_{d_{5}} / Y_{t}$. When $Y_{d_{5}} / Y_{t} \geq 0.5$, the theoretical predictions on $R_{\gamma \gamma}, R_{V V^{*}}$ exceed the experimental range in Eq.(40), and the numerical evaluations on $m_{A^{0}}, m_{H^{0}}$ are below $600 \mathrm{GeV}$. 


\section{SUMMARY}

In framework of the BLMSSM, we attempt to account for the experimental data on Higgs reported by ATLAS and CMS recently. Assuming the Yukawa couplings between Higgs doublets and exotic quarks satisfying $Y_{u_{4}}, Y_{d_{5}}<Y_{t}$ as well as $Y_{d_{4}}, Y_{u_{5}}<Y_{b}$, we find the theoretical predictions on $R_{\gamma \gamma}, R_{V V^{*}}$ fitting the experimental data in Eq. (40) very well when $m_{h_{0}}=125.9 \mathrm{GeV}$. Furthermore, the numerical evaluations on $m_{A^{0}}, m_{H^{0}}$ exceed $600 \mathrm{GeV}$ simultaneously in some parameter space of the BLMSSM.

\section{Acknowledgments}

The work has been supported by the National Natural Science Foundation of China (NNSFC) with Grant No. 11275036, No. 11047002 and Natural Science Fund of Hebei University with Grant No. 2011JQ05, No. 2012-242.

\section{Appendix A: The couplings between neutral Higgs and exotic quarks}

In the mass basis, the couplings between the neutral Higgs and charged $2 / 3$ exotic quarks are written as

$$
\begin{aligned}
\mathcal{L}_{H t^{\prime} t^{\prime}}=\frac{1}{\sqrt{2}} & \sum_{i, j=1}^{2}\left\{\left[Y_{u_{4}}\left(W_{t}^{\dagger}\right)_{i 2}\left(U_{t}\right)_{1 j} \cos \alpha+Y_{u_{5}}\left(W_{t}^{\dagger}\right)_{i 1}\left(U_{t}\right)_{2 j} \sin \alpha\right] h^{0} \bar{t}_{i+3} P_{L} t_{j+3}\right. \\
& +\left[Y_{u_{4}}\left(U_{t}^{\dagger}\right)_{i 1}\left(W_{t}\right)_{2 j} \cos \alpha+Y_{u_{5}}\left(U_{t}^{\dagger}\right)_{i 2}\left(W_{t}\right)_{1 j} \sin \alpha\right] h^{0} \bar{t}_{i+3} P_{R} t_{j+3} \\
& +\left[Y_{u_{4}}\left(W_{t}^{\dagger}\right)_{i 2}\left(U_{t}\right)_{1 j} \sin \alpha-Y_{u_{5}}\left(W_{t}^{\dagger}\right)_{i 1}\left(U_{t}\right)_{2 j} \cos \alpha\right] H^{0} \bar{t}_{i+3} P_{L} t_{j+3} \\
& \left.+\left[Y_{u_{4}}\left(U_{t}^{\dagger}\right)_{i 1}\left(W_{t}\right)_{2 j} \sin \alpha-Y_{u_{5}}\left(U_{t}^{\dagger}\right)_{i 2}\left(W_{t}\right)_{1 j} \cos \alpha\right] H^{0} \bar{t}_{i+3} P_{R} t_{j+3}\right\} \\
& +\frac{i}{\sqrt{2}} \sum_{i, j=1}^{2}\left\{\left[Y_{u_{4}}\left(W_{t}^{\dagger}\right)_{i 2}\left(U_{t}\right)_{1 j} \cos \beta+Y_{u_{5}}\left(W_{t}^{\dagger}\right)_{i 1}\left(U_{t}\right)_{2 j} \sin \beta\right] A^{0} \bar{t}_{i+3} P_{L} t_{j+3}\right. \\
& -\left[Y_{u_{4}}\left(U_{t}^{\dagger}\right)_{i 1}\left(W_{t}\right)_{2 j} \cos \beta+Y_{u_{5}}\left(U_{t}^{\dagger}\right)_{i 2}\left(W_{t}\right)_{1 j} \sin \beta\right] A^{0} \bar{t}_{i+3} P_{R} t_{j+3} \\
& +\left[Y_{u_{4}}\left(W_{t}^{\dagger}\right)_{i 2}\left(U_{t}\right)_{1 j} \sin \beta-Y_{u_{5}}\left(W_{t}^{\dagger}\right)_{i 1}\left(U_{t}\right)_{2 j} \cos \beta\right] G^{0} \bar{t}_{i+3} P_{L} t_{j+3} \\
& \left.-\left[Y_{u_{4}}\left(U_{t}^{\dagger}\right)_{i 1}\left(W_{t}\right)_{2 j} \sin \beta-Y_{u_{5}}\left(U_{t}^{\dagger}\right)_{i 2}\left(W_{t}\right)_{1 j} \cos \beta\right] G^{0} \bar{t}_{i+3} P_{R} t_{j+3}\right\} \\
& -\frac{1}{\sqrt{2}} \sum_{i, j=1}^{2}\left\{\left[\lambda_{u}\left(W_{t}^{\dagger}\right)_{i 2}\left(U_{t}\right)_{2 j} \cos \alpha_{B}-\lambda_{Q}\left(W_{t}^{\dagger}\right)_{i 1}\left(U_{t}\right)_{1 j} \sin \alpha_{B}\right] h_{B}^{0} \bar{t}_{i+3} P_{L} t_{j+3}\right.
\end{aligned}
$$




$$
\begin{aligned}
& +\left[\lambda_{u}\left(U_{t}^{\dagger}\right)_{i 2}\left(W_{t}\right)_{2 j} \cos \alpha_{B}-\lambda_{Q}\left(U_{t}^{\dagger}\right)_{i 2}\left(W_{t}\right)_{2 j} \sin \alpha_{B}\right] h_{B}^{0} \bar{t}_{i+3} P_{R} t_{j+3} \\
& +\left[\lambda_{u}\left(W_{t}^{\dagger}\right)_{i 2}\left(U_{t}\right)_{2 j} \sin \alpha_{B}+\lambda_{Q}\left(W_{t}^{\dagger}\right)_{i 1}\left(U_{t}\right)_{1 j} \cos \alpha_{B}\right] H_{B}^{0} \bar{t}_{i+3} P_{L} t_{j+3} \\
& \left.+\left[\lambda_{u}\left(U_{t}^{\dagger}\right)_{i 2}\left(W_{t}\right)_{2 j} \sin \alpha_{B}+\lambda_{Q}\left(U_{t}^{\dagger}\right)_{i 2}\left(W_{t}\right)_{2 j} \cos \alpha_{B}\right] H_{B}^{0} \bar{t}_{i+3} P_{R} t_{j+3}\right\} \\
& -\frac{i}{\sqrt{2}} \sum_{i, j=1}^{2}\left\{\left[\lambda_{u}\left(W_{t}^{\dagger}\right)_{i 2}\left(U_{t}\right)_{2 j} \cos \beta_{B}-\lambda_{Q}\left(W_{t}^{\dagger}\right)_{i 1}\left(U_{t}\right)_{1 j} \sin \beta_{B}\right] A_{B}^{0} \bar{t}_{i+3} P_{L} t_{j+3}\right. \\
& -\left[\lambda_{u}\left(U_{t}^{\dagger}\right)_{i 2}\left(W_{t}\right)_{2 j} \cos \beta_{B}-\lambda_{Q}\left(U_{t}^{\dagger}\right)_{i 2}\left(W_{t}\right)_{2 j} \sin \beta_{B}\right] A_{B}^{0} \bar{t}_{i+3} P_{R} t_{j+3} \\
& +\left[\lambda_{u}\left(W_{t}^{\dagger}\right)_{i 2}\left(U_{t}\right)_{2 j} \sin \beta_{B}+\lambda_{Q}\left(W_{t}^{\dagger}\right)_{i 1}\left(U_{t}\right)_{1 j} \cos \beta_{B}\right] G_{B}^{0} \bar{t}_{i+3} P_{L} t_{j+3} \\
& \left.-\left[\lambda_{u}\left(U_{t}^{\dagger}\right)_{i 2}\left(W_{t}\right)_{2 j} \sin \beta_{B}+\lambda_{Q}\left(U_{t}^{\dagger}\right)_{i 2}\left(W_{t}\right)_{2 j} \cos \beta_{B}\right] H_{B}^{0} \bar{t}_{i+3} P_{R} t_{j+3}\right\}
\end{aligned}
$$

Similarly, the couplings between the neutral Higgs and charged $-1 / 3$ exotic quarks are written as

$$
\begin{aligned}
& \mathcal{L}_{H b^{\prime} b^{\prime}}=\frac{1}{\sqrt{2}} \sum_{i, j=1}^{2}\left\{\left[Y_{d_{4}}\left(W_{b}^{\dagger}\right)_{i 2}\left(U_{b}\right)_{1 j} \sin \alpha-Y_{d_{5}}\left(W_{b}^{\dagger}\right)_{i 1}\left(U_{b}\right)_{2 j} \cos \alpha\right] h^{0} \bar{b}_{i+3} P_{L} b_{j+3}\right. \\
& +\left[Y_{d_{4}}\left(U_{b}^{\dagger}\right)_{i 1}\left(W_{b}\right)_{2 j} \sin \alpha-Y_{d_{5}}\left(U_{b}^{\dagger}\right)_{i 2}\left(W_{b}\right)_{1 j} \cos \alpha\right] h^{0} \bar{b}_{i+3} P_{R} b_{j+3} \\
& -\left[Y_{d_{4}}\left(W_{b}^{\dagger}\right)_{i 2}\left(U_{b}\right)_{1 j} \cos \alpha+Y_{d_{5}}\left(W_{b}^{\dagger}\right)_{i 1}\left(U_{b}\right)_{2 j} \sin \alpha\right] H^{0} \bar{b}_{i+3} P_{L} b_{j+3} \\
& -\left[Y_{d_{4}}\left(U_{b}^{\dagger}\right)_{i 1}\left(W_{b}\right)_{2 j} \cos \alpha+Y_{d_{5}}\left(U_{b}^{\dagger}\right)_{i 2}\left(W_{b}\right)_{1 j} \sin \alpha\right] H^{0} \bar{b}_{i+3} P_{R} b_{j+3} \\
& +\frac{i}{\sqrt{2}} \sum_{i, j=1}^{2}\left\{\left[Y_{d_{4}}\left(W_{b}^{\dagger}\right)_{i 2}\left(U_{b}\right)_{1 j} \sin \beta-Y_{d_{5}}\left(W_{b}^{\dagger}\right)_{i 1}\left(U_{b}\right)_{2 j} \cos \beta\right] A^{0} \bar{b}_{i+3} P_{L} b_{j+3}\right. \\
& -\left[Y_{d_{4}}\left(U_{b}^{\dagger}\right)_{i 1}\left(W_{b}\right)_{2 j} \sin \beta-Y_{d_{5}}\left(U_{b}^{\dagger}\right)_{i 2}\left(W_{b}\right)_{1 j} \cos \beta\right] A^{0} \bar{b}_{i+3} P_{R} b_{j+3} \\
& -\left[Y_{d_{4}}\left(W_{b}^{\dagger}\right)_{i 2}\left(U_{b}\right)_{1 j} \cos \beta+Y_{d_{5}}\left(W_{b}^{\dagger}\right)_{i 1}\left(U_{b}\right)_{2 j} \sin \beta\right] G^{0} \bar{b}_{i+3} P_{L} b_{j+3} \\
& +\left[Y_{d_{4}}\left(U_{b}^{\dagger}\right)_{i 1}\left(W_{b}\right)_{2 j} \cos \beta+Y_{d_{5}}\left(U_{b}^{\dagger}\right)_{i 2}\left(W_{b}\right)_{1 j} \sin \beta\right] G^{0} \bar{b}_{i+3} P_{R} b_{j+3} \\
& -\frac{1}{\sqrt{2}} \sum_{i, j=1}^{2}\left\{\left[\lambda_{d}\left(W_{b}^{\dagger}\right)_{i 2}\left(U_{b}\right)_{2 j} \cos \alpha_{B}+\lambda_{Q}\left(W_{b}^{\dagger}\right)_{i 1}\left(U_{b}\right)_{1 j} \sin \alpha_{B}\right] h_{B}^{0} \bar{b}_{i+3} P_{L} b_{j+3}\right. \\
& +\left[\lambda_{d}\left(U_{b}^{\dagger}\right)_{i 2}\left(W_{b}\right)_{2 j} \cos \alpha_{B}+\lambda_{Q}\left(U_{b}^{\dagger}\right)_{i 2}\left(W_{b}\right)_{2 j} \sin \alpha_{B}\right] h_{B}^{0} \bar{b}_{i+3} P_{R} b_{j+3} \\
& +\left[\lambda_{d}\left(W_{b}^{\dagger}\right)_{i 2}\left(U_{b}\right)_{2 j} \sin \alpha_{B}+\lambda_{Q}\left(W_{b}^{\dagger}\right)_{i 1}\left(U_{b}\right)_{1 j} \cos \alpha_{B}\right] H_{B}^{0} \bar{b}_{i+3} P_{L} b_{j+3} \\
& \left.+\left[\lambda_{d}\left(U_{b}^{\dagger}\right)_{i 2}\left(W_{b}\right)_{2 j} \sin \alpha_{B}+\lambda_{Q}\left(U_{b}^{\dagger}\right)_{i 2}\left(W_{b}\right)_{2 j} \cos \alpha_{B}\right] H_{B}^{0} \bar{b}_{i+3} P_{R} b_{j+3}\right\} \\
& -\frac{i}{\sqrt{2}} \sum_{i, j=1}^{2}\left\{\left[\lambda_{d}\left(W_{b}^{\dagger}\right)_{i 2}\left(U_{b}\right)_{2 j} \cos \beta_{B}+\lambda_{Q}\left(W_{b}^{\dagger}\right)_{i 1}\left(U_{b}\right)_{1 j} \sin \beta_{B}\right] A_{B}^{0} \bar{b}_{i+3} P_{L} b_{j+3}\right. \\
& -\left[\lambda_{d}\left(U_{b}^{\dagger}\right)_{i 2}\left(W_{b}\right)_{2 j} \cos \beta_{B}+\lambda_{Q}\left(U_{b}^{\dagger}\right)_{i 2}\left(W_{b}\right)_{2 j} \sin \beta_{B}\right] A_{B}^{0} \bar{b}_{i+3} P_{R} b_{j+3} \\
& +\left[\lambda_{d}\left(W_{b}^{\dagger}\right)_{i 2}\left(U_{b}\right)_{2 j} \sin \beta_{B}+\lambda_{Q}\left(W_{b}^{\dagger}\right)_{i 1}\left(U_{b}\right)_{1 j} \cos \beta_{B}\right] G_{B}^{0} \bar{b}_{i+3} P_{L} b_{j+3}
\end{aligned}
$$




$$
\left.-\left[\lambda_{d}\left(U_{b}^{\dagger}\right)_{i 2}\left(W_{b}\right)_{2 j} \sin \beta_{B}+\lambda_{Q}\left(U_{b}^{\dagger}\right)_{i 2}\left(W_{b}\right)_{2 j} \cos \beta_{B}\right] G_{B}^{0} \bar{b}_{i+3} P_{R} b_{j+3}\right\}
$$

\section{Appendix B: mass squared matrices for exotic squarks}

For charged 2/3 exotic scalar quarks, the elements of mass squared matrix are written as

$$
\begin{aligned}
\mathcal{M}_{\tilde{t}^{\prime}}^{2}\left(\tilde{Q}_{4}^{1 *} \tilde{Q}_{4}^{1}\right)= & m_{\tilde{Q}_{4}}^{2}+\frac{1}{2} Y_{u_{4}}^{2} v_{u}^{2}+\frac{1}{2} Y_{d_{4}}^{2} v_{d}^{2}+\frac{1}{2} \lambda_{Q}^{2} v_{B}^{2}+\left(\frac{1}{2}-\frac{2}{3} s_{\mathrm{w}}^{2}\right) m_{\mathrm{z}}^{2} \cos 2 \beta \\
& +\frac{B_{4}}{2} m_{z_{B}}^{2} \cos 2 \beta_{B}, \\
\mathcal{M}_{\tilde{t}^{\prime}}^{2}\left(\tilde{U}_{4}^{c} \tilde{U}_{4}^{c *}\right)= & m_{\tilde{U}_{4}}^{2}+\frac{1}{2} Y_{u_{4}}^{2} v_{u}^{2}+\frac{1}{2} \lambda_{u}^{2} \bar{v}_{B}^{2}-\frac{2}{3} s_{\mathrm{w}}^{2} m_{\mathrm{z}}^{2} \cos 2 \beta-\frac{B_{4}}{2} m_{z_{B}}^{2} \cos 2 \beta_{B}, \\
\mathcal{M}_{\tilde{t}^{\prime}}^{2}\left(\tilde{Q}_{5}^{2 c} \tilde{Q}_{5}^{2 c *}\right)= & m_{\tilde{Q}_{5}}^{2}+\frac{1}{2} Y_{u_{5}}^{2} v_{d}^{2}+\frac{1}{2} Y_{d_{5}}^{2} v_{u}^{2}+\frac{1}{2} \lambda_{Q}^{2} v_{B}^{2}+\left(\frac{1}{2}-\frac{1}{3} s_{\mathrm{w}}^{2}\right) m_{\mathrm{z}}^{2} \cos 2 \beta \\
& -\frac{1+B_{4}}{2} m_{z_{B}}^{2} \cos 2 \beta_{B}, \\
\mathcal{M}_{\tilde{t}^{\prime}}^{2}\left(\tilde{U}_{5}^{*} \tilde{U}_{5}\right)= & m_{\tilde{U}_{5}}^{2}+\frac{1}{2} Y_{u_{5}}^{2} v_{d}^{2}+\frac{1}{2} \lambda_{u}^{2} \bar{v}_{B}^{2}+\frac{2}{3} s_{\mathrm{w}}^{2} m_{\mathrm{z}}^{2} \cos 2 \beta+\frac{1+B_{4}}{2} m_{z_{B}}^{2} \cos 2 \beta_{B}, \\
\mathcal{M}_{\tilde{t}^{\prime}}^{2}\left(\tilde{U}_{4}^{c} \tilde{Q}_{4}^{1}\right)= & -\frac{1}{\sqrt{2}} v_{u} Y_{u_{4}} A_{u_{4}}+\frac{1}{\sqrt{2}} Y_{u_{4}} \mu v_{d}, \\
\mathcal{M}_{\tilde{t}^{\prime}}^{2}\left(\tilde{Q}_{5}^{2 c} \tilde{Q}_{4}^{1}\right)= & -\frac{1}{\sqrt{2}} v_{B} \lambda_{Q} A_{B Q}+\sqrt{2} \lambda_{Q} \mu_{B} \bar{v}_{B}, \\
\mathcal{M}_{\tilde{t}^{\prime}}^{2}\left(\tilde{U}_{5}^{*} \tilde{Q}_{4}^{1}\right)= & -\frac{1}{\sqrt{2}} Y_{u_{4}} \lambda_{u} v_{u} \bar{v}_{B}+\frac{1}{\sqrt{2}} Y_{u_{5}} \lambda_{Q} v_{d} v_{B}, \\
\mathcal{M}_{\tilde{t}^{\prime}}^{2}\left(\tilde{Q}_{5}^{2 c} \tilde{U}_{4}^{c *}\right)= & \frac{1}{2} \lambda_{Q} Y_{u_{4}} v_{u} v_{B}-\frac{1}{2} \lambda_{u} Y_{u_{5}} v_{d} \bar{v}_{B}, \\
\mathcal{M}_{\tilde{t}^{\prime}}^{2}\left(\tilde{U}_{5}^{*} \tilde{U}_{4}^{c *}\right)= & -\frac{1}{\sqrt{2}} \lambda_{u} A_{B U} \bar{v}_{B}+\frac{1}{\sqrt{2}} \lambda_{u} \mu_{B} v_{B}, \\
\mathcal{M}_{\tilde{t}^{\prime}}^{2}\left(\tilde{Q}_{5}^{2 c} \tilde{U}_{5}\right)= & -\frac{1}{\sqrt{2}} Y_{u_{5}} A_{u_{5}} v_{d}+\frac{1}{\sqrt{2}} Y_{u_{5}} \mu v_{u} .
\end{aligned}
$$

For charged $-1 / 3$ exotic scalar quarks, the elements of mass squared matrix are given as

$$
\begin{aligned}
\mathcal{M}_{\tilde{t}^{\prime}}^{2}\left(\tilde{Q}_{4}^{2 *} \tilde{Q}_{4}^{2}\right)= & m_{\tilde{Q}_{4}}^{2}+\frac{1}{2} Y_{u_{4}}^{2} v_{u}^{2}+\frac{1}{2} Y_{d_{4}}^{2} v_{d}^{2}+\frac{1}{2} \lambda_{Q}^{2} v_{B}^{2}-\left(\frac{1}{2}-\frac{2}{3} s_{\mathrm{W}}^{2}\right) m_{\mathrm{z}}^{2} \cos 2 \beta \\
& +\frac{B_{4}}{2} m_{z_{B}}^{2} \cos 2 \beta_{B}, \\
\mathcal{M}_{\tilde{t}^{\prime}}^{2}\left(\tilde{D}_{4}^{c} \tilde{D}_{4}^{c *}\right)= & m_{\tilde{D}_{4}}^{2}+\frac{1}{2} Y_{d_{4}}^{2} v_{d}^{2}+\frac{1}{2} \lambda_{d}^{2} \bar{v}_{B}^{2}-\frac{1}{3} s_{\mathrm{W}}^{2} m_{\mathrm{z}}^{2} \cos 2 \beta-\frac{B_{4}}{2} m_{z_{B}}^{2} \cos 2 \beta_{B}, \\
\mathcal{M}_{\tilde{t}^{\prime}}^{2}\left(\tilde{Q}_{5}^{1 c} \tilde{Q}_{5}^{1 c *}\right)= & m_{\tilde{Q}_{5}}^{2}+\frac{1}{2} Y_{u_{5}}^{2} v_{d}^{2}+\frac{1}{2} Y_{d_{5}}^{2} v_{u}^{2}+\frac{1}{2} \lambda_{Q}^{2} v_{B}^{2}-\left(\frac{1}{2}+\frac{1}{3} s_{\mathrm{W}}^{2}\right) m_{\mathrm{z}}^{2} \cos 2 \beta
\end{aligned}
$$




$$
\begin{aligned}
&-\frac{1+B_{4}}{2} m_{Z_{B}}^{2} \cos 2 \beta_{B} \\
& \mathcal{M}_{\tilde{t}^{\prime}}^{2}\left(\tilde{D}_{5}^{*} \tilde{D}_{5}\right)=m_{\tilde{D}_{5}}^{2}+\frac{1}{2} Y_{d_{5}}^{2} v_{u}^{2}+\frac{1}{2} \lambda_{d}^{2} \bar{v}_{B}^{2}+\frac{1}{3} s_{\mathrm{w}}^{2} m_{\mathrm{z}}^{2} \cos 2 \beta+\frac{1+B_{4}}{2} m_{z_{B}}^{2} \cos 2 \beta_{B} \\
& \mathcal{M}_{\tilde{t}^{\prime}}^{2}\left(\tilde{D}_{4}^{c} \tilde{Q}_{4}^{2}\right)=-\frac{1}{\sqrt{2}} Y_{d_{4}} v_{d} A_{d_{4}}+\frac{1}{\sqrt{2}} Y_{d_{4}} \mu v_{d} \\
& \mathcal{M}_{\tilde{t}^{\prime}}^{2}\left(\tilde{Q}_{5}^{1 c} \tilde{Q}_{4}^{2}\right)=-\frac{1}{\sqrt{2}} \lambda_{Q} v_{B} A_{B Q}+\sqrt{2} \lambda_{Q} \mu_{B} \bar{v}_{B} \\
& \mathcal{M}_{\tilde{t}^{\prime}}^{2}\left(\tilde{D}_{5}^{*} \tilde{Q}_{4}^{2}\right)=-\frac{1}{\sqrt{2}} Y_{d_{4}} \lambda_{d} v_{d} \bar{v}_{B}+\frac{1}{\sqrt{2}} Y_{d_{5}} \lambda_{Q} v_{u} v_{B} \\
& \mathcal{M}_{\tilde{t}^{\prime}}^{2}\left(\tilde{Q}_{5}^{1 c} \tilde{D}_{4}^{c *}\right)=\frac{1}{2} \lambda_{Q} Y_{d_{4}} v_{d} v_{B}+\frac{1}{2} \lambda_{d} Y_{d_{5}} v_{u} \bar{v}_{B} \\
& \mathcal{M}_{\tilde{t}^{\prime}}^{2}\left(\tilde{D}_{5}^{*} \tilde{D}_{4}^{c *}\right)=-\frac{1}{\sqrt{2}} \lambda_{d} A_{B D} \bar{v}_{B}+\frac{1}{\sqrt{2}} \lambda_{d} \mu_{B} v_{B} \\
& \mathcal{M}_{\tilde{t}^{\prime}}^{2}\left(\tilde{Q}_{5}^{1 c} \tilde{D}_{5}\right)=-\frac{1}{\sqrt{2}} Y_{d_{5}} A_{d_{5}} v_{u}+\frac{1}{\sqrt{2}} Y_{d_{5}} \mu v_{d}
\end{aligned}
$$

\section{Appendix C: The couplings between neutral Higgs and exotic squarks}

In the mass basis, the couplings between the neutral Higgs and exotic squarks are

$$
\begin{aligned}
& \mathcal{L}_{H \tilde{\mathcal{U}}_{i}^{*} \tilde{\mathcal{U}}_{j}}=\sum_{i, j}^{4}\left\{\left[\xi_{u i j}^{S} \cos \alpha-\xi_{d i j}^{S} \sin \alpha\right] h^{0} \tilde{\mathcal{U}}_{i}^{*} \tilde{\mathcal{U}}_{j}+\left[\eta_{u i j}^{S} \cos \alpha-\eta_{d i j}^{S} \sin \alpha\right] h^{0} \tilde{\mathcal{D}}_{i}^{*} \tilde{\mathcal{D}}_{j}\right. \\
& +\left[\xi_{u i j}^{S} \sin \alpha+\xi_{d i j}^{S} \cos \alpha\right] H^{0} \tilde{\mathcal{U}}_{i}^{*} \tilde{\mathcal{U}}_{j}+\left[\eta_{u i j}^{S} \sin \alpha+\eta_{d i j}^{S} \cos \alpha\right] H^{0} \tilde{\mathcal{D}}_{i}^{*} \tilde{\mathcal{D}}_{j} \\
& +i\left[\xi_{u i j}^{P} \cos \beta-\xi_{d i j}^{P} \sin \beta\right] A^{0} \tilde{\mathcal{U}}_{i}^{*} \tilde{\mathcal{U}}_{j}+i\left[\eta_{u i j}^{P} \cos \beta-\eta_{d i j}^{P} \sin \beta\right] A^{0} \tilde{\mathcal{D}}_{i}^{*} \tilde{\mathcal{D}}_{j} \\
& +i\left[\xi_{u i j}^{P} \sin \beta+\xi_{d i j}^{P} \cos \beta\right] G^{0} \tilde{\mathcal{U}}_{i}^{*} \tilde{\mathcal{U}}_{j}+i\left[\eta_{u i j}^{P} \sin \beta+\eta_{d i j}^{P} \cos \beta\right] G^{0} \tilde{\mathcal{D}}_{i}^{*} \tilde{\mathcal{D}}_{j} \\
& +\left[\varsigma_{u i j}^{S} \cos \alpha_{B}-\varsigma_{d i j}^{S} \sin \alpha_{B}\right] h_{B}^{0} \tilde{\mathcal{U}}_{i}^{*} \tilde{\mathcal{U}}_{j}+\left[\zeta_{u i j}^{S} \cos \alpha_{B}-\zeta_{d i j}^{S} \sin \alpha_{B}\right] h_{B}^{0} \tilde{\mathcal{D}}_{i}^{*} \tilde{\mathcal{D}}_{j} \\
& +\left[\varsigma_{u i j}^{S} \sin \alpha_{B}+\varsigma_{d i j}^{S} \cos \alpha_{B}\right] H_{B}^{0} \tilde{\mathcal{U}}_{i}^{*} \tilde{\mathcal{U}}_{j}+\left[\zeta_{u i j}^{S} \sin \alpha_{B}+\zeta_{d i j}^{S} \cos \alpha_{B}\right] H_{B}^{0} \tilde{\mathcal{D}}_{i}^{*} \tilde{\mathcal{D}}_{j} \\
& +i\left[\varsigma_{u i j}^{P} \cos \beta_{B}-\varsigma_{d i j}^{P} \sin \beta_{B}\right] A_{B}^{0} \tilde{\mathcal{U}}_{i}^{*} \tilde{\mathcal{U}}_{j}+i\left[\zeta_{u i j}^{P} \cos \beta_{B}-\zeta_{d i j}^{P} \sin \beta_{B}\right] A_{B}^{0} \tilde{\mathcal{D}}_{i}^{*} \tilde{\mathcal{D}}_{j} \\
& \left.+i\left[\varsigma_{u i j}^{P} \sin \beta_{B}+\varsigma_{d i j}^{P} \cos \beta_{B}\right] G_{B}^{0} \tilde{\mathcal{U}}_{i}^{*} \tilde{\mathcal{U}}_{j}+i\left[\zeta_{u i j}^{P} \sin \beta_{B}+\zeta_{d i j}^{P} \cos \beta_{B}\right] G_{B}^{0} \tilde{\mathcal{D}}_{i}^{*} \tilde{\mathcal{D}}_{j}\right\}
\end{aligned}
$$

with

$$
\begin{aligned}
\xi_{u i j}^{S}= & \frac{1}{\sqrt{2}} Y_{u_{5}} \mu\left(U_{i 3}^{\dagger} U_{4 j}+U_{i 4}^{\dagger} U_{3 j}\right)+\frac{1}{2} \lambda_{Q} Y_{u_{4}} v_{B}\left(U_{i 3}^{\dagger} U_{2 j}+U_{i 2}^{\dagger} U_{3 j}\right) \\
& -\frac{1}{2} \lambda_{u} Y_{u_{4}} \bar{v}_{B}\left(U_{i 1}^{\dagger} U_{4 j}+U_{i 4}^{\dagger} U_{1 j}\right)+\frac{e^{2}}{4 s_{\mathrm{w}}^{2}} v_{u}\left(U_{i 3}^{\dagger} U_{3 j}-U_{i 1}^{\dagger} U_{1 j}\right)
\end{aligned}
$$




$$
\begin{aligned}
& +\frac{e^{2}}{12 c_{\mathrm{W}}^{2}} v_{u}\left(U_{i 1}^{\dagger} U_{1 j}-U_{i 3}^{\dagger} U_{3 j}-4 U_{i 2}^{\dagger} U_{2 j}+4 U_{i 4}^{\dagger} U_{4 j}\right) \\
& -\frac{1}{\sqrt{2}} A_{u_{4}} Y_{u_{4}}\left(U_{i 2}^{\dagger} U_{1 j}+U_{i 1}^{\dagger} U_{2 j}\right) \\
& \xi_{d i j}^{S}=\frac{1}{\sqrt{2}} Y_{u_{4}} \mu\left(U_{i 2}^{\dagger} U_{1 j}+U_{i 1}^{\dagger} U_{2 j}\right)+\frac{1}{2} \lambda_{Q} Y_{u_{5}} v_{B}\left(U_{i 5}^{\dagger} U_{1 j}+U_{i 1}^{\dagger} U_{5 j}\right) \\
& -\frac{1}{2} \lambda_{u} Y_{u_{5}} \bar{v}_{B}\left(U_{i 2}^{\dagger} U_{3 j}+U_{i 3}^{\dagger} U_{2 j}\right)-\frac{e^{2}}{4 s_{\mathrm{w}}^{2}} v_{d}\left(U_{i 3}^{\dagger} U_{3 j}+U_{i 1}^{\dagger} U_{1 j}\right) \\
& -\frac{e^{2}}{12 c_{\mathrm{W}}^{2}} v_{d}\left(U_{i 1}^{\dagger} U_{1 j}-U_{i 3}^{\dagger} U_{3 j}-4 U_{i 2}^{\dagger} U_{2 j}+4 U_{i 4}^{\dagger} U_{4 j}\right) \\
& -\frac{1}{\sqrt{2}} A_{u_{5}} Y_{u_{5}}\left(U_{i 3}^{\dagger} U_{4 j}+U_{i 4}^{\dagger} U_{3 j}\right) \\
& \eta_{u i j}^{S}=\frac{1}{\sqrt{2}} Y_{d_{4}} \mu\left(D_{i 2}^{\dagger} D_{1 j}+D_{i 1}^{\dagger} D_{2 j}\right)+\frac{1}{2} \lambda_{Q} Y_{d_{5}} v_{B}\left(D_{i 4}^{\dagger} D_{1 j}+D_{i 1}^{\dagger} D_{4 j}\right) \\
& -\frac{1}{2} \lambda_{d} Y_{d_{5}} \bar{v}_{B}\left(D_{i 2}^{\dagger} D_{3 j}+D_{i 3}^{\dagger} D_{2 j}\right)+\frac{e^{2}}{4 s_{\mathrm{w}}^{2}} v_{u}\left(D_{i 1}^{\dagger} D_{1 j}-D_{i 3}^{\dagger} D_{3 j}\right) \\
& +\frac{e^{2}}{12 c_{\mathrm{W}}^{2}} v_{u}\left(D_{i 1}^{\dagger} D_{1 j}-D_{i 3}^{\dagger} D_{3 j}+2 D_{i 2}^{\dagger} D_{2 j}-2 D_{i 4}^{\dagger} D_{4 j}\right) \\
& -\frac{1}{\sqrt{2}} A_{d_{5}} Y_{d_{5}}\left(D_{i 3}^{\dagger} D_{4 j}+D_{i 4}^{\dagger} D_{3 j}\right) \\
& \eta_{d i j}^{S}=\frac{1}{\sqrt{2}} Y_{d_{5}} \mu\left(D_{i 3}^{\dagger} D_{4 j}+D_{i 4}^{\dagger} D_{3 j}\right)+\frac{1}{2} \lambda_{Q} Y_{d_{4}} v_{B}\left(D_{i 3}^{\dagger} D_{2 j}+D_{i 2}^{\dagger} D_{3 j}\right) \\
& -\frac{1}{2} \lambda_{d} Y_{d_{4}} \bar{v}_{B}\left(D_{i 1}^{\dagger} D_{4 j}+D_{i 4}^{\dagger} D_{1 j}\right)-\frac{e^{2}}{4 s_{\mathrm{w}}^{2}} v_{d}\left(D_{i 1}^{\dagger} D_{1 j}-D_{i 3}^{\dagger} D_{3 j}\right) \\
& -\frac{e^{2}}{12 c_{\mathrm{W}}^{2}} v_{u}\left(D_{i 1}^{\dagger} D_{1 j}-D_{i 3}^{\dagger} D_{3 j}+2 D_{i 2}^{\dagger} D_{2 j}-2 D_{i 4}^{\dagger} D_{4 j}\right) \\
& -\frac{1}{\sqrt{2}} A_{d_{4}} Y_{d_{4}}\left(D_{i 2}^{\dagger} D_{1 j}+D_{i 1}^{\dagger} D_{2 j}\right) \\
& \xi_{u i j}^{P}=\frac{1}{\sqrt{2}} Y_{u_{5}} \mu\left(U_{i 3}^{\dagger} U_{4 j}-U_{i 4}^{\dagger} U_{3 j}\right)-\frac{1}{2} \lambda_{Q} Y_{u_{4}} v_{B}\left(U_{i 3}^{\dagger} U_{2 j}-U_{i 2}^{\dagger} U_{3 j}\right) \\
& +\frac{1}{2} \lambda_{u} Y_{u_{4}} \bar{v}_{B}\left(U_{i 1}^{\dagger} U_{4 j}-U_{i 4}^{\dagger} U_{1 j}\right)-\frac{1}{\sqrt{2}} A_{u_{4}} Y_{u_{4}}\left(U_{i 2}^{\dagger} U_{1 j}-U_{i 1}^{\dagger} U_{2 j}\right), \\
& \xi_{d i j}^{P}=\frac{1}{\sqrt{2}} Y_{u_{4}} \mu\left(U_{i 2}^{\dagger} U_{1 j}-U_{i 1}^{\dagger} U_{2 j}\right)-\frac{1}{2} \lambda_{Q} Y_{u_{5}} v_{B}\left(U_{i 5}^{\dagger} U_{1 j}-U_{i 1}^{\dagger} U_{5 j}\right) \\
& +\frac{1}{2} \lambda_{u} Y_{u_{5}} \bar{v}_{B}\left(U_{i 2}^{\dagger} U_{3 j}-U_{i 3}^{\dagger} U_{2 j}\right)-\frac{1}{\sqrt{2}} A_{u_{5}} Y_{u_{5}}\left(U_{i 3}^{\dagger} U_{4 j}-U_{i 4}^{\dagger} U_{3 j}\right) \text {, } \\
& \eta_{u i j}^{P}=\frac{1}{\sqrt{2}} Y_{d_{4}} \mu\left(D_{i 2}^{\dagger} D_{1 j}-D_{i 1}^{\dagger} D_{2 j}\right)-\frac{1}{2} \lambda_{Q} Y_{d_{5}} v_{B}\left(D_{i 4}^{\dagger} D_{1 j}-D_{i 1}^{\dagger} D_{4 j}\right)
\end{aligned}
$$




$$
\begin{aligned}
& +\frac{1}{2} \lambda_{d} Y_{d_{5}} \bar{v}_{B}\left(D_{i 2}^{\dagger} D_{3 j}-D_{i 3}^{\dagger} D_{2 j}\right)-\frac{1}{\sqrt{2}} A_{d_{5}} Y_{d_{5}}\left(D_{i 3}^{\dagger} D_{4 j}-D_{i 4}^{\dagger} D_{3 j}\right), \\
& \eta_{d i j}^{P}=\frac{1}{\sqrt{2}} Y_{d_{5}} \mu\left(D_{i 3}^{\dagger} D_{4 j}-D_{i 4}^{\dagger} D_{3 j}\right)-\frac{1}{2} \lambda_{Q} Y_{d_{4}} v_{B}\left(D_{i 3}^{\dagger} D_{2 j}-D_{i 2}^{\dagger} D_{3 j}\right) \\
& +\frac{1}{2} \lambda_{d} Y_{d_{4}} \bar{v}_{B}\left(D_{i 1}^{\dagger} D_{4 j}-D_{i 4}^{\dagger} D_{1 j}\right)-\frac{1}{\sqrt{2}} A_{d_{4}} Y_{d_{4}}\left(D_{i 2}^{\dagger} D_{1 j}-D_{i 1}^{\dagger} D_{2 j}\right) \text {, } \\
& \varsigma_{u i j}^{S}=\frac{1}{\sqrt{2}} \lambda_{u} \mu_{B}\left(U_{i 2}^{\dagger} U_{4 j}+U_{i 4}^{\dagger} U_{2 j}\right)+\frac{1}{2} \lambda_{Q} Y_{u_{4}} v_{u}\left(U_{i 3}^{\dagger} U_{2 j}+U_{i 2}^{\dagger} U_{3 j}\right) \\
& -\frac{1}{2} \lambda_{Q} Y_{u_{5}} v_{d}\left(U_{i 4}^{\dagger} U_{1 j}+U_{i 1}^{\dagger} U_{4 j}\right)+g_{B}^{2} v_{B}\left(B_{4} U_{i 1}^{\dagger} U_{1 j}-\left(1+B_{4}\right) U_{i 3}^{\dagger} U_{3 j}\right. \\
& \left.-B_{4} U_{i 2}^{\dagger} U_{2 j}+\left(1+B_{4}\right) U_{i 4}^{\dagger} U_{4 j}\right)-\frac{1}{\sqrt{2}} A_{B Q} \lambda_{Q}\left(U_{i 3}^{\dagger} U_{1 j}+U_{i 1}^{\dagger} U_{3 j}\right) \text {, } \\
& \varsigma_{d i j}^{S}=\frac{1}{\sqrt{2}} \lambda_{Q} \mu_{B}\left(U_{i 3}^{\dagger} U_{1 j}+U_{i 1}^{\dagger} U_{3 j}\right)-\frac{1}{2} \lambda_{u} Y_{u_{4}} v_{u}\left(U_{i 1}^{\dagger} U_{4 j}+U_{i 4}^{\dagger} U_{1 j}\right) \\
& -\frac{1}{2} \lambda_{u} Y_{u 5} v_{d}\left(U_{i 2}^{\dagger} U_{3 j}+U_{i 3}^{\dagger} U_{2 j}\right)-g_{B}^{2} \bar{v}_{B}\left(B_{4} U_{i 1}^{\dagger} U_{1 j}-\left(1+B_{4}\right) U_{i 3}^{\dagger} U_{3 j}\right. \\
& \left.-B_{4} U_{i 2}^{\dagger} U_{2 j}+\left(1+B_{4}\right) U_{i 4}^{\dagger} U_{4 j}\right)+\frac{1}{\sqrt{2}} A_{B U} \lambda_{u}\left(U_{i 2}^{\dagger} U_{4 j}+U_{i 4}^{\dagger} U_{2 j}\right) \text {, } \\
& \zeta_{u i j}^{S}=\frac{1}{\sqrt{2}} \lambda_{d} \mu_{B}\left(D_{i 2}^{\dagger} D_{4 j}+D_{i 4}^{\dagger} D_{2 j}\right)+\frac{1}{2} \lambda_{Q} Y_{d_{4}} v_{d}\left(D_{i 3}^{\dagger} D_{2 j}+D_{i 2}^{\dagger} D_{3 j}\right) \\
& -\frac{1}{2} \lambda_{Q} Y_{d_{5}} v_{u}\left(D_{i 4}^{\dagger} D_{1 j}+D_{i 1}^{\dagger} D_{4 j}\right)+g_{B}^{2} v_{B}\left(B_{4} D_{i 1}^{\dagger} D_{1 j}-\left(1+B_{4}\right) D_{i 3}^{\dagger} D_{3 j}\right. \\
& \left.-B_{4} D_{i 2}^{\dagger} D_{2 j}+\left(1+B_{4}\right) D_{i 4}^{\dagger} D_{4 j}\right)-\frac{1}{\sqrt{2}} A_{B Q} \lambda_{Q}\left(D_{i 3}^{\dagger} D_{1 j}+D_{i 1}^{\dagger} D_{3 j}\right) \text {, } \\
& \zeta_{d i j}^{S}=-\frac{1}{\sqrt{2}} \lambda_{Q} \mu_{B}\left(D_{i 3}^{\dagger} D_{1 j}+D_{i 1}^{\dagger} D_{3 j}\right)-\frac{1}{2} \lambda_{d} Y_{d_{4}} v_{d}\left(D_{i 1}^{\dagger} D_{4 j}+D_{i 4}^{\dagger} D_{1 j}\right) \\
& -\frac{1}{2} \lambda_{d} Y_{d_{5}} v_{u}\left(D_{i 2}^{\dagger} D_{3 j}+D_{i 3}^{\dagger} D_{2 j}\right)-g_{B}^{2} \bar{v}_{B}\left(B_{4} D_{i 1}^{\dagger} D_{1 j}-\left(1+B_{4}\right) D_{i 3}^{\dagger} D_{3 j}\right. \\
& \left.-B_{4} D_{i 2}^{\dagger} D_{2 j}+\left(1+B_{4}\right) D_{i 4}^{\dagger} D_{4 j}\right)+\frac{1}{\sqrt{2}} A_{B D} \lambda_{d}\left(D_{i 2}^{\dagger} D_{4 j}+D_{i 4}^{\dagger} D_{2 j}\right) \text {, } \\
& \varsigma_{u i j}^{P}=\frac{1}{\sqrt{2}} \lambda_{u} \mu_{B}\left(U_{i 2}^{\dagger} U_{4 j}-U_{i 4}^{\dagger} U_{2 j}\right)+\frac{1}{2} \lambda_{Q} Y_{u_{4}} v_{u}\left(U_{i 3}^{\dagger} U_{2 j}-U_{i 2}^{\dagger} U_{3 j}\right) \\
& -\frac{1}{2} \lambda_{Q} Y_{u_{5}} v_{d}\left(U_{i 4}^{\dagger} U_{1 j}-U_{i 1}^{\dagger} U_{4 j}\right)-\frac{1}{\sqrt{2}} A_{B Q} \lambda_{Q}\left(U_{i 3}^{\dagger} U_{1 j}-U_{i 1}^{\dagger} U_{3 j}\right), \\
& \varsigma_{d i j}^{P}=\frac{1}{\sqrt{2}} \lambda_{Q} \mu_{B}\left(U_{i 3}^{\dagger} U_{1 j}-U_{i 1}^{\dagger} U_{3 j}\right)-\frac{1}{2} \lambda_{u} Y_{u_{4}} v_{u}\left(U_{i 1}^{\dagger} U_{4 j}-U_{i 4}^{\dagger} U_{1 j}\right) \\
& -\frac{1}{2} \lambda_{u} Y_{u_{5}} v_{d}\left(U_{i 2}^{\dagger} U_{3 j}-U_{i 3}^{\dagger} U_{2 j}\right)+\frac{1}{\sqrt{2}} A_{B U} \lambda_{u}\left(U_{i 2}^{\dagger} U_{4 j}-U_{i 4}^{\dagger} U_{2 j}\right) \text {, } \\
& \zeta_{u i j}^{P}=\frac{1}{\sqrt{2}} \lambda_{d} \mu_{B}\left(D_{i 2}^{\dagger} D_{4 j}-D_{i 4}^{\dagger} D_{2 j}\right)+\frac{1}{2} \lambda_{Q} Y_{d_{4}} v_{d}\left(D_{i 3}^{\dagger} D_{2 j}-D_{i 2}^{\dagger} D_{3 j}\right)
\end{aligned}
$$




$$
\begin{aligned}
& -\frac{1}{2} \lambda_{Q} Y_{d_{5}} v_{u}\left(D_{i 4}^{\dagger} D_{1 j}-D_{i 1}^{\dagger} D_{4 j}\right)-\frac{1}{\sqrt{2}} A_{B Q} \lambda_{Q}\left(D_{i 3}^{\dagger} D_{1 j}-D_{i 1}^{\dagger} D_{3 j}\right), \\
\zeta_{d i j}^{P}= & -\frac{1}{\sqrt{2}} \lambda_{Q} \mu_{B}\left(D_{i 3}^{\dagger} D_{1 j}-D_{i 1}^{\dagger} D_{3 j}\right)-\frac{1}{2} \lambda_{d} Y_{d_{4}} v_{d}\left(D_{i 1}^{\dagger} D_{4 j}-D_{i 4}^{\dagger} D_{1 j}\right) \\
& -\frac{1}{2} \lambda_{d} Y_{d_{5}} v_{u}\left(D_{i 2}^{\dagger} D_{3 j}-D_{i 3}^{\dagger} D_{2 j}\right)+\frac{1}{\sqrt{2}} A_{B D} \lambda_{d}\left(D_{i 2}^{\dagger} D_{4 j}-D_{i 4}^{\dagger} D_{2 j}\right) .
\end{aligned}
$$

\section{Appendix D: The radiative corrections to the mass squared matrix from exotic}

\section{lepton fields}

$$
\begin{aligned}
& \Delta_{11}^{L}=\frac{G_{F} m_{\nu_{4}}^{4}}{\sqrt{2} \pi^{2} \sin ^{2} \beta} \cdot \frac{\mu^{2}\left(A_{\nu_{4}}-\mu \cot \beta\right)^{2}}{\left(m_{\tilde{\nu}_{4}^{1}}^{2}-m_{\tilde{\nu}_{4}^{2}}^{2}\right)^{2}} g\left(m_{\tilde{\nu}_{4}^{1}}, m_{\tilde{\nu}_{4}^{2}}\right) \\
& +\frac{G_{F} m_{e_{4}}^{4}}{\sqrt{2} \pi^{2} \cos ^{2} \beta}\left\{\ln \frac{m_{\tilde{e}_{4}^{1}} m_{\tilde{e}_{4}^{2}}}{m_{e_{4}}^{2}}+\frac{A_{e_{4}}\left(A_{e_{4}}-\mu \tan \beta\right)}{m_{\tilde{e}_{4}^{1}}^{2}-m_{\tilde{e}_{4}^{2}}^{2}} \ln \frac{m_{\tilde{e}_{4}^{1}}^{2}}{m_{\tilde{e}_{4}^{2}}^{2}}\right. \\
& \left.+\frac{A_{e_{4}}^{2}\left(A_{e_{4}}-\mu \tan \beta\right)^{2}}{\left(m_{\tilde{e}_{4}^{1}}^{2}-m_{\tilde{e}_{4}^{2}}^{2}\right)^{2}} g\left(m_{\tilde{e}_{4}^{1}}, m_{\tilde{e}_{4}^{2}}\right)\right\} \\
& +\frac{G_{F} m_{\nu_{5}}^{4}}{\sqrt{2} \pi^{2} \cos ^{2} \beta}\left\{\ln \frac{m_{\tilde{\nu}_{5}^{1}} m_{\tilde{\nu}_{5}^{2}}}{m_{\nu_{5}}^{2}}+\frac{A_{\nu_{5}}\left(A_{\nu_{5}}-\mu \tan \beta\right)}{m_{\tilde{\nu}_{5}^{1}}^{2}-m_{\tilde{\nu}_{5}^{2}}^{2}} \ln \frac{m_{\tilde{\nu}_{5}^{1}}^{2}}{m_{\tilde{\nu}_{5}^{2}}^{2}}\right. \\
& \left.+\frac{A_{\nu_{5}}^{2}\left(A_{\nu_{5}}-\mu \tan \beta\right)^{2}}{\left(m_{\tilde{\nu}_{5}^{1}}^{2}-m_{\tilde{\nu}_{5}^{2}}^{2}\right)^{2}} g\left(m_{\tilde{\nu}_{5}^{1}}, m_{\tilde{\nu}_{5}^{2}}\right)\right\} \\
& +\frac{G_{F} m_{e_{5}}^{4}}{\sqrt{2} \pi^{2} \sin ^{2} \beta} \cdot \frac{\mu^{2}\left(A_{e_{5}}-\mu \cot \beta\right)^{2}}{\left(m_{\tilde{e}_{5}^{1}}^{2}-m_{\tilde{e}_{5}^{2}}^{2}\right)^{2}} g\left(m_{\tilde{e}_{5}^{1}}, m_{\tilde{e}_{5}^{2}}\right), \\
& \Delta_{12}^{L}=\frac{G_{F} m_{\nu_{4}}^{4}}{2 \sqrt{2} \pi^{2} \sin ^{2} \beta} \cdot \frac{\mu\left(-A_{\nu_{4}}+\mu \cot \beta\right)}{m_{\tilde{\nu}_{4}^{1}}^{2}-m_{\tilde{\nu}_{4}^{2}}^{2}}\left\{\ln \frac{m_{\tilde{\nu}_{4}^{1}}}{m_{\tilde{\nu}_{4}^{2}}}+\frac{A_{\nu_{4}}\left(A_{\nu_{4}}-\mu \cot \beta\right)}{m_{\tilde{\nu}_{4}^{1}}^{2}-m_{\tilde{\nu}_{4}^{2}}^{2}} g\left(m_{\tilde{\nu}_{4}^{1}}, m_{\tilde{\nu}_{4}^{2}}\right)\right\} \\
& +\frac{G_{F} m_{e_{4}}^{4}}{2 \sqrt{2} \pi^{2} \cos ^{2} \beta} \cdot \frac{\mu\left(-A_{e_{4}}+\mu \tan \beta\right)}{m_{\tilde{e}_{4}^{1}}^{2}-m_{\tilde{e}_{4}^{2}}^{2}}\left\{\ln \frac{m_{\tilde{e}_{4}^{1}}}{m_{\tilde{e}_{4}^{2}}}+\frac{A_{e_{4}}\left(A_{e_{4}}-\mu \tan \beta\right)}{m_{\tilde{e}_{4}^{1}}^{2}-m_{\tilde{e}_{4}^{2}}^{2}} g\left(m_{\tilde{e}_{4}^{1}}, m_{\tilde{e}_{4}^{2}}\right)\right\} \\
& +\frac{G_{F} m_{\nu_{5}}^{4}}{2 \sqrt{2} \pi^{2} \cos ^{2} \beta} \cdot \frac{\mu\left(-A_{\nu_{5}}+\mu \tan \beta\right)}{m_{\tilde{\nu}_{5}^{1}}^{2}-m_{\tilde{\nu}_{5}^{2}}^{2}}\left\{\ln \frac{m_{\tilde{\nu}_{5}^{1}}}{m_{\tilde{\nu}_{5}^{2}}}+\frac{A_{\nu_{5}}\left(A_{\nu_{5}}-\mu \tan \beta\right)}{m_{\tilde{\nu}_{5}^{1}}^{2}-m_{\tilde{\nu}_{5}^{2}}^{2}} g\left(m_{\tilde{\nu}_{5}^{1}}, m_{\tilde{\nu}_{5}^{2}}\right)\right\} \\
& +\frac{G_{F} m_{e_{5}}^{4}}{2 \sqrt{2} \pi^{2} \sin ^{2} \beta} \cdot \frac{\mu\left(-A_{e_{5}}+\mu \cot \beta\right)}{m_{\tilde{e}_{5}^{1}}^{2}-m_{\tilde{e}_{5}^{2}}^{2}}\left\{\ln \frac{m_{\tilde{e}_{5}^{1}}}{m_{\tilde{e}_{5}^{2}}}+\frac{A_{e_{5}}\left(A_{e_{5}}-\mu \cot \beta\right)}{m_{\tilde{e}_{5}^{1}}^{2}-m_{\tilde{e}_{5}^{2}}^{2}} g\left(m_{\tilde{e}_{5}^{1}}, m_{\tilde{e}_{5}^{2}}\right)\right\},
\end{aligned}
$$




$$
\begin{aligned}
\Delta_{22}^{L}= & \frac{G_{F} m_{\nu_{4}}^{4}}{\sqrt{2} \pi^{2} \sin ^{2} \beta}\left\{\ln \frac{m_{\tilde{\nu}_{4}^{1}} m_{\tilde{\nu}_{4}^{2}}}{m_{\nu_{4}}^{2}}+\frac{A_{\nu_{4}}\left(A_{\nu_{4}}-\mu \cot \beta\right)}{m_{\tilde{\nu}_{4}^{1}}^{2}-m_{\tilde{\nu}_{4}^{2}}^{2}} \ln \frac{m_{\tilde{\nu}_{4}^{1}}^{2}}{m_{\tilde{\nu}_{4}^{2}}^{2}}\right. \\
& \left.+\frac{A_{\nu_{4}}^{2}\left(A_{\nu_{4}}-\mu \cot \beta\right)^{2}}{\left(m_{\tilde{\nu}_{4}^{1}}^{2}-m_{\tilde{\nu}_{4}^{2}}^{2}\right)^{2}} g\left(m_{\tilde{\nu}_{4}^{1}}, m_{\tilde{\nu}_{4}^{2}}\right)\right\} \\
& +\frac{G_{F} m_{e_{4}}^{4}}{\sqrt{2} \pi^{2} \cos ^{2} \beta} \cdot \frac{\mu^{2}\left(A_{e_{4}}-\mu \tan \beta\right)^{2}}{\left(m_{\tilde{e}_{4}^{1}}^{2}-m_{\tilde{e}_{4}^{2}}^{2}\right)^{2}} g\left(m_{\tilde{e}_{4}^{1}}, m_{\tilde{e}_{4}^{2}}\right) \\
& +\frac{G_{F} m_{\nu_{5}}^{4}}{\sqrt{2} \pi^{2} \cos ^{2} \beta} \cdot \frac{\mu^{2}\left(A_{\nu_{5}}-\mu \tan \beta\right)^{2}}{\left(m_{\tilde{\nu}_{5}^{1}}^{2}-m_{\tilde{\nu}_{5}^{2}}^{2}\right)^{2}} g\left(m_{\tilde{\nu}_{5}^{1}}, m_{\tilde{\nu}_{5}^{2}}\right) \\
& +\frac{G_{F} m_{e_{5}}^{4}}{\sqrt{2} \pi^{2} \sin ^{2} \beta}\left\{\ln \frac{m_{\tilde{e}_{5}^{1}} m_{\tilde{e}_{5}^{2}}}{m_{e_{5}}^{2}}+\frac{A_{e_{5}}\left(A_{e_{5}}-\mu \cot \beta\right)}{m_{\tilde{e}_{5}^{1}}^{2}-m_{\tilde{e}_{5}^{2}}^{2}} \ln \frac{m_{\tilde{e}_{5}^{1}}^{2}}{m_{\tilde{e}_{5}^{2}}^{2}}\right. \\
& \left.+\frac{A_{e_{5}}^{2}\left(A_{e_{5}}-\mu \cot \beta\right)^{2}}{\left(m_{\tilde{e}_{5}^{1}}^{2}-m_{\tilde{e}_{5}^{2}}^{2}\right)^{2}} g\left(m_{\tilde{e}_{5}^{1}}, m_{\tilde{e}_{5}^{2}}\right)\right\}
\end{aligned}
$$

[1] CMS Collaboration, Phys. Lett. B716(2012)30.

[2] ATLAS Collaboration, Phys. Lett. B716(2012)1.

[3] H. E. Haber and G. L. Kane, Phys. Rep. 117(1985)75; J. Rosiek, Phys. Rev. D41(1990)3464.

[4] P. Minkoski, Phys. Lett.B67(1977)421; T. Yanagida, in Proceedings of the Workshop on the Unified Theory and the Baryon Number in the Universe, edited by O. Sawada et.al. (KEK, Tsukuba, 1979), p. 95; M. Gell-Mann, P. Ramond, and R. Slansky, in Supergravity, edited by P. van Nieuwenhuizen et.al. (North-Holland, Amsterdam, 1979), p315; S. L. Glashow, in Quarks and Leptons, Cargése, edited by M. Lévy et.al. (Plenum, New York, 1980), p707; R. N. Mohapatra and G. Senjanovic, Phys. Rev. Lett. 44(1980)912.

[5] P. F. Perez, Phys. Lett. B711(2012)353.

[6] J. M. Arnold, P. F. Perez, B. Fornal, and S. Spinner, Phys. Rev. D85(2012)115024.

[7] P. F. Perez and M. B. Wise, JHEP1108(2011)068; Phys. Rev. D82(2010)011901; ibid.84(2011)055015; T. R. Dulaney, P. F. Perez and M. B. Wise, Phys. Rev. D83(2011)023520.

[8] H. E. Haber, R. Hempfling, Phys. Rev. Lett. 66(1991)1815. 
[9] S. Heinemeyer, W. Hollik and G. Weiglein, Comput. Phys. Commun. 24(2000)76; Eur. Phys. J. C9(1999)343; G. Degrassi, S. Heinemeyer, W. Hollik, P. Slavich and G. Weiglein, Eur. Phys. J. C28(2003)133; M. Frank, T. Hahn, S. Heinemeyer, W. Hollik, H. Rzehak and G. Weiglein, JHEP0702(2007)047.

[10] Y. Okada, M. Yamaguchi and T. Yanagida, Prog. Theor. Phys.85(1991)1; J. R. Ellis, G. Ridolfi and F. Zwirner, Phys. Lett. B257(1991)83; ibid.262(1991)477; S. P. Li and M. Sher, Phys. Lett. B140(1984)33; R. Barbieri and M. Frigeni, Phys. Lett. B258(1991)395; M. Drees and M. M. Nojiri, Phys. Rev. D45(1992)2482; J. A. Casas, J. R. Espinosa, M. Quiros and A. Riotto, Nucl. Phys. B436(1995)3[Erratum-ibid. B439(1995)466]; M. A. Diaz and H. E. Haber, Phys. Rev. D46(1992)3086; M. S. Carena, M. Quiros and C. E. M. Wagner, Nucl. Phys. B461(1996)407.

[11] A. Arbey, M. Battaglia, A. Djouadi and F. Mahmoudi, JHEP1209(2012)107.

[12] C. Anastasiou and K. Melnikov, Nucl. Phys. B646(2002)220.

[13] J. R. Ellis, M. K. Gaillard and D. V. Nanopoulos, Nucl. Phys. B106(1976)292; M. A. Shifman, A. I. Vainshtein, M. B. Voloshin and V. I. Zakharov, Sov. J. Nucl. Phys. 30(1979)711; A. Djouadi, Phys. Rept. 459(2008)1; J. F. Gunion, H. E. Haber, G. L. Kane and S. Dawson, The Higgs Hunters Guide, Addison-Wesley, Reading (USA), 1990; M. Carena, I. Low and C. E. M. Wagner, arXiv:1206.1082 [hep-ph].

[14] W.-Y. Keung, W. J. Marciano, Phys. Rev. D30(1984)248; J. F. Gunion, H. E. Haber, G. Kane, S. Dawson, The Higgs Hunter's Guide, Perseus Books(1990).

[15] P. Gonzáleza, S. Palmerb, M. Wiebuschc, K. Williamsd, arXiv:1211.3079[hep-ph]; W. Bernreuther, P. Gonzalez, M. Wiebusch, Eur. Phys. J. C69 (2010) 31;

[16] J. Beringer et al.(Particle Data Group), Phys. Rev. D86(2012)010001. 